\title{
Sequential Colimits in Homotopy Type Theory
}

\author{
Kristina Sojakova \\ Cornell University \\ USA \\ ks858@cornell.edu
}

\author{
Floris van Doorn \\ University of Pittsburgh \\ USA \\ fpvdoorn@gmail.com
}

\author{
Egbert Rijke \\ University of Ljubljana \\ Slovenia \\ e.m.rijke@gmail.com
}

\begin{abstract}
Sequential colimits are an important class of higher inductive types. We present a self-contained and fully formalized proof of the conjecture that in homotopy type theory sequential colimits appropriately commute with $\Sigma$-types. This result allows us to give short proofs of a number of useful corollaries, some of which were conjectured in other works: the commutativity of sequential colimits with identity types, with homotopy fibers, loop spaces, and truncations, and the preservation of the properties of truncatedness and connectedness under sequential colimits. Our entire development carries over to $(\infty, 1)$-toposes using Shulman's recent interpretation of homotopy type theory into these structures.
\end{abstract}

\section{CCS Concepts: • Theory of computation $\rightarrow$ Type the-} ory.

Keywords: sequential colimits, higher inductive types, homotopy type theory

\section{ACM Reference Format:}

Kristina Sojakova, Floris van Doorn, and Egbert Rijke. 2020. Sequential Colimits in Homotopy Type Theory. In Proceedings of the 35th Annual ACM/IEEE Symposium on Logic in Computer Science (LICS '20), Fuly 8-11, 2020, Saarbrücken, Germany. ACM, New York, NY, USA, 14 pages. https://doi.org/10.1145/3373718.3394801

\section{Introduction}

Homotopy type theory (HoTT) [The Univalent Foundations Program, Institute for Advanced Study 2013] is an extension of Martin-Löf's dependent type theory [Martin-Löf 1975], in which types are thought of as spaces, dependent types as fibrations, terms of types as points of those spaces, and terms of the identity type $x=y$ for any $x, y: A$ as paths in $A$ from $x$ to $y$. This is the so-called homotopy interpretation of type theory, see [Awodey and Warren 2009]. Two further ingredients are then added to this type theory: the univalence

Permission to make digital or hard copies of all or part of this work for personal or classroom use is granted without fee provided that copies are not made or distributed for profit or commercial advantage and that copies bear this notice and the full citation on the first page. Copyrights for components of this work owned by others than ACM must be honored. Abstracting with credit is permitted. To copy otherwise, or republish, to post on servers or to redistribute to lists, requires prior specific permission and/or a fee. Request permissions from permissions@acm.org.

LICS '20, July 8-11, 2020, Saarbrücken, Germany

(c) 2020 Association for Computing Machinery.

ACM ISBN 978-1-4503-7104-9/20/07 . \$15.00

https://doi.org/10.1145/3373718.3394801 axiom due to Voevodsky [Kapulkin and Lumsdaine 2012; Voevodsky 2010, 2011] and higher inductive types due to Bauer, Lumsdaine, Shulman, and Warren [Awodey et al. 2011; Lumsdaine 2011; Lumsdaine and Shulman 2017; Shulman 2011]. The univalence axiom characterizes the identity type of the universe, and higher inductive types allow (among other things) constructions of new types by specifying their points and identifications between those points, in a fashion similar to introducing new algebras by generators and relations. Indeed, Sojakova [2016] showed that a large class of higher inductive types arise as homotopy initial algebras. A semantics of higher inductive types suitable for interpreting the development in this paper is given in [Lumsdaine and Shulman 2017].

An important class of examples of higher inductive types are homotopy colimits, such as pushouts, coequalizers, and sequential colimits. Sequential colimits in homotopy type theory were first studied by Brunerie [2016], who showed that the infinite dimensional sphere, defined as the colimit of the finite dimensional spheres, is contractible. A sequential colimit is the homotopy colimit of a diagram of the form

$$
\mathrm{A}(0) \stackrel{\mathrm{a}(0)}{\longrightarrow} \mathrm{A}(1) \stackrel{\mathrm{a}(1)}{\longrightarrow} \mathrm{A}(2) \stackrel{\mathrm{a}(2)}{\longrightarrow} \cdots
$$

Many interesting examples of types can be presented as sequential colimits. For example, van Doorn [2016] showed that the propositional truncation of a type can be obtained as a sequential colimit. Brunerie [2016] performed the James construction in HoTT, showing that the loop space of a suspension can be presented as a sequential colimit. Rijke [2017] showed that $n$-truncations can be obtained as sequential colimits, via a construction that was generalized in [Christensen et al. 2018] to arbitrary reflective subuniverses. Moreover, stable homotopy groups and homology groups can be defined using a sequential colimit [Graham 2017]. We expect many more interesting applications to follow. For example, Shulman [2013] suggested that the spectrification of a prespectrum can be presented as a sequential colimit, although a proof that the resulting pre-spectrum is indeed a spectrum is still work in progress.

Moreover, many constructions in the semantics of programming languages take the form of sequential colimits. For example, the existence of a certain class of inductive types is guaranteed by a theorem of [Adamek 1974], which shows that if an endofunctor preserves sequential colimits, then initial algebras for this functor exist and can themselves be characterized as sequential colimits. Sequential colimits 
are also ubiquitous in domain theory: for instance, Scott's $D_{\infty}$ model of the untyped $\lambda$-calculus can be seen as a sequential colimit of embeddings (chapter 18, page 480 of [Barendregt 1985]). We hope that the results in the present paper can be used to develop the semantics of programming languages internally in homotopy type theory, thereby also imbuing these classic results with an $\infty$-categorical twist.

In HoTT, the sequential colimit of a sequence as in Diagram 1 is defined as a higher inductive type called $A_{\infty}$. The point constructor of $A_{\infty}$ is

$$
\iota: \Pi_{n: \mathbb{N}} \mathrm{A}(n) \rightarrow \mathrm{A}_{\infty}
$$

We note that we use a convention where $\rightarrow$ binds stronger than $\Pi$ (we use a similar convention for $\Sigma$ ). The only path constructor of $A_{\infty}$ is

$$
\kappa: \Pi_{n: \mathbb{N}} \Pi_{x: \mathrm{A}(n)} \iota(n+1, \mathrm{a}(n, x))=\iota(n, x)
$$

Just like all higher inductive types, the sequential colimit comes equipped with an induction principle and computation rules. Those principles essentially ensure that the type $A_{\infty}$ indeed behaves as the homotopy colimit of the sequence $\mathrm{A}(0) \rightarrow \mathrm{A}(1) \rightarrow \cdots$. In fact, the induction principle implies a dependent universal property.

To explain our main technical result, we consider the situation where we are given a type family $\mathrm{B}(n): \mathrm{A}(n) \rightarrow \mathrm{U}$ over each $\mathrm{A}(n)$, equipped with functions $\mathrm{b}(n, a): \mathrm{B}(n, a) \rightarrow$ $\mathrm{B}(n+1, \mathrm{a}(n, a))$ for each $a: \mathrm{A}(n)$. We picture this situation as follows:

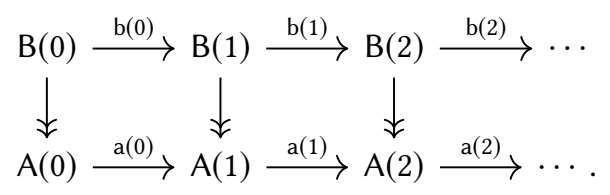

Here the double arrows indicate that the type family $\mathrm{B}(n)$ is fibered over $\mathrm{A}(n)$. Now we can consider the sequence of total spaces (i.e. $\sum$-types)

$$
\Sigma_{a: \mathrm{A}(0)} \mathrm{B}(0, a) \longrightarrow \Sigma_{a: \mathrm{A}(1)} \mathrm{B}(1, a) \longrightarrow \cdots
$$

and we write $\operatorname{colim}(\Sigma(\mathrm{A}, \mathrm{B}))$ for its homotopy colimit. On the other hand, we can form a type family $B_{\infty}: A_{\infty} \rightarrow U$ and compare its total space with colim $\left(\sum(\mathrm{A}, \mathrm{B})\right)$. To construct $\mathrm{B}_{\infty}$, we first form for each $a: \mathrm{A}(n)$ the sequence

$$
\mathrm{B}(n, a) \longrightarrow \mathrm{B}(n+1, \mathrm{a}(n, a)) \longrightarrow \cdots
$$

and write $\mathrm{B}_{\infty}(n, a)$ its colimit. Now we can construct an equivalence $\mathrm{B}_{\infty}(n, a) \simeq \mathrm{B}_{\infty}(n+1, \mathrm{a}(n, a))$ for any $a: \mathrm{A}(n)$, so by univalence we obtain a type family $B_{\infty}: A_{\infty} \rightarrow U$ as a result. Our main technical theorem, Theorem 5.1, states that we have a commuting triangle

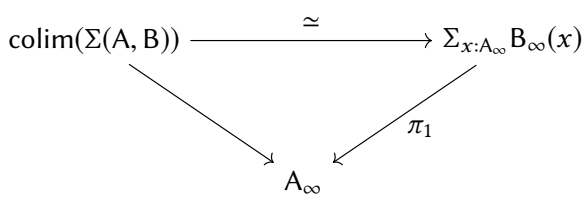

in which the top map is an equivalence. Among the chief consequences of our theorem (see section 7) is the characterization of the identity type of a sequential colimit as a sequential colimit of identity types. This corollary can be seen as complementing the results of Kraus and von Raumer [2019], who characterize the identity types of coequalizers and pushouts.

Related Work. Our result has been conjectured for the setting of homotopy type theory by E. Rijke in 2015. The proof we give here, and the accompanying formalization, is the original one with which we settled the conjecture for homotopy type theory in July of 2017. Seven months later, R. Bocquet gave an alternative formalization ${ }^{1}$ of our main result that reasons about sequences and colimits in a stream-like fashion reminiscent of coinduction. A disadvantage of this technique is that certain crucial definitions become much more complicated than necessary. For instance, in our development the canonical map from the colimit of the sum to the sum of colimits is easy to define (see Theorem 5.1); in particular, it maps each canonical element $\iota(n,(a, b))$ to a pair of canonical elements $(\iota(n, a), \iota(0, b))$. In the stream-like presentation, however, even the mapping of canonical elements already requires a significant amount of path algebra. This is not optimal since from the user's point of view, it is the usability of the canonical map that matters rather than the particulars of how this map is shown to be an equivalence (as the latter is a mere proposition).

In 2017 Bocquet gave a formalization ${ }^{2}$ of the analogous result for a different type theory - a version of cubical type theory - where he represents sequential colimits as an indexed higher inductive type. This indexed HIT has constructors $\iota(a)$ and $\kappa(a)$ for $a: A(0)$ (i.e., not for a general $n: \mathbb{N}$ but only for the base case $n:=0$ ), plus an additional point constructor lift that takes an element $x: \operatorname{colim}(n \mapsto \mathrm{A}(n+1), n \mapsto \mathrm{a}(n+1))$ of the colimit of the lifted sequence that drops the first index, and produces a canonical element of the colimit of the original sequence.

We do not wish to use indexed HITs for the following reasons: they are not a standard feature of "book" HoTT; their formal theory has not yet been fully developed; and as our work shows, they are unnecessary for establishing the result. We likewise wish to use homotopy type theory as opposed to a version of cubical type theory for several reasons: the former has a known interpretation into $(\infty, 1)$ toposes due to [Shulman 2019]; cubical type theories are not

\footnotetext{
${ }^{1}$ https://github.com/RafaelBocquet/CoinductiveSequentialColimits

${ }^{2}$ https://github.com/mortberg/cubicaltt/blob/seqcolim/examples/ seqcolim.ctt
} 
yet known to be conservative over homotopy type theory; we prefer to keep the assumptions on the underlying type theory minimal; and since cubical type theories "interpret" homotopy type theory, our proof can readily be adapted to any of these alternative systems.

Comparison with Classical Results. A classical result in 1-category theory states that filtered colimits over small filtered categories commute with finite limits (see [MacLane 1971] or [Borceux 1994]). A generalization of this result to the $\infty$-categorical setting can be found in [Lurie 2009]: Proposition 5.3.3.3 implies that colimits over any $\kappa$-filtered category commute with $\kappa$-small limits in homotopy types, and Example 7.3.4.7 uses this result to establish that colimits over any small filtered $(\infty, 1)$-category into an $(\infty, 1)$-topos commute with finite limits. Our development of sequential colimits is completely formalized, requires very little prior setup, and relies exclusively on suitable induction principles. This is in the spirit of homotopy type theory, which allows type theorists with little knowledge of category theory to not only understand but also to prove results from higher category theory in an elementary fashion. Shulman's recent interpretation of homotopy type theory into $(\infty, 1)$-topos guarantees that our results hold in any $(\infty, 1)$-topos.

As a conceptual contribution, we present our proof in a fashion that aims to minimize the need for the explicit manipulation of paths and relies instead on appropriate generalizations. As a prime example of our technique, we describe the following trick: when establishing the equivalence between $\operatorname{colim}(\Sigma(\mathrm{A}, \mathrm{B}))$ and $\sum_{x: \mathrm{A}_{\infty}} \mathrm{B}_{\infty}(x)$, instead of directly using the provided induction principles on the sum of two sequential colimits - which is a nested induction with point-point, pointpath, path-point, and path-path cases - we prove instead that the latter type satisfies the much easier induction principle of the former type. The desired equivalence then follows immediately.

Both the original formalization ${ }^{3}$ as well as the cleaned up version ${ }^{4}$ use the proof assistant Lean $2^{5}$. Lean 2 is an older version of Lean ${ }^{6}$ that offers significantly more support for HoTT [de Moura et al. 2015].

Overview. In Section 2 we briefly review some homotopy type theory; in Section 3 we establish some basic properties of sequences and colimits; in Section 4 we discuss fibered sequences and construct the type family $B_{\infty}$; in Section 5 we state our main result and show that it follows from a more convenient induction principle on $\sum_{x: \mathrm{A}_{\infty}} \mathrm{B}_{\infty}(x)$; in Section 6 we sketch the proof of the aforementioned induction principle; and in Section 7 we give some important corollaries of our main result.

\footnotetext{
${ }^{3}$ https://github.com/EgbertRijke/sequential_colimits/tree/ 0da6c4c0f42ff9292fe815e21b97a5a56060e45f

${ }^{4}$ https://github.com/cmu-phil/Spectral/tree/master/colimit

${ }^{5} \mathrm{https} / / /$ github.com/leanprover/lean2

${ }^{6}$ https://leanprover.github.io/
}

\section{Homotopy Type Theory}

We recall some basic notions and constructions from homotopy type theory; for a full account of the theory, see [The Univalent Foundations Program, Institute for Advanced Study 2013]. We distinguish two forms of equality in homotopy type theory: judgmental equality and typal equality. Judgmental equality is used for computation. When two terms $t_{1}$ and $t_{2}$ of type $A$ are syntactically equal, we write $t_{1} \equiv t_{2}$. For example, $1+1 \equiv 2$ of type $\mathbb{N}$. The second form of equality is typal equality, which is introduced in the form of Martin-Löf's identity type. For any two terms $x$ and $y$ of type $A$ there is a type $x=y$, and proving that $x=y$ holds amounts to constructing a term of the type $x=y$, which is called a path. The constant path from $x$ to itself is called refl $x$. For any path $p: x=y$ we write $p^{-1}: y=x$ for its inverse, and for any two paths $p: x=y$ and $q: y=z$, we write $p \cdot q$ for their concatenation. Concatenation is defined in such a way that refl $I_{x} \cdot \operatorname{refl}_{x} \equiv \operatorname{refl}_{x}$, and there is an identification $(p \cdot q) \cdot r=p \cdot(q \cdot r)$ for any three concatenatable paths. Moreover, any function $f: A \rightarrow B$ respects paths: for any path $p: x=y$ in $A$ there is a path $\operatorname{ap}_{f}(p): f(x)=f(y)$.

We recall that a homotopy between two (dependent) maps $f, g: \Pi_{x: A} B(x)$ is a term of type $f \sim g:=\Pi_{x: A} f(x)=g(x)$. An equivalence is then defined as a map $f: A \rightarrow B$ equipped with a right and a left inverse, i.e., functions $g: B \rightarrow A$ and $h: B \rightarrow A$ with homotopies $f \circ g \sim \operatorname{id}_{B}$ and $h \circ f \sim \operatorname{id}_{A}$. We write $A \simeq B$ for the type of equivalences from $A$ to $B$. The univalence axiom asserts that the canonical map $(A=B) \rightarrow$ $(A \simeq B)$ is an equivalence. We will write $\bar{p}: A \rightarrow B$ for the function associated to a path $p: A=B$ in $\mathrm{U}$. The function extensionality principle (which follows from univalence) states that for any $f, g: \Pi_{x: A} B(x)$, the map $(f=g) \rightarrow(f \sim$ $g$ ) is an equivalence. For $f: A \rightarrow B$ and $y: B$, the homotopy fiber of $f$ at $y$ is defined $\operatorname{asib}_{f}(y):=\left(\sum_{x: A} f(x)=y\right)$.

An important concept in homotopy type theory is that of transport. Given a type family $B: A \rightarrow \mathrm{U}$ and a path $p: x=y$ in $A$, we have a function $p \#_{B}: B(x) \rightarrow B(y)$, with refl $_{x} \#_{B} \equiv \mathrm{id}$. Using transport, we can also show that dependent functions respect paths: for any dependent function $f: \Pi_{x: A} B(x)$ and any path $p: x=y$ in $A$, we can't directly compare $f(x)$ of type $B(x)$ with $f(y)$ of type $B(y)$, but there is a path $\operatorname{apd}_{f}(p): p \#_{B} f(x)=f(y)$ in $B(y)$.

The notion that a type $A$ is $n$-truncated is defined by recursion on $n: \mathbb{Z}, n \geq-2$. We say that $A$ is (-2)-truncated or contractible if we can find a inhabitant of $\sum_{x: A} \Pi_{y: A} x=y$. The type $A$ is $(n+1)$-truncated if for all $x, y: A$ the type $x=y$ is $n$-truncated. For any type $A$ we write $\|A\|_{n}$ for its $n$-truncation, i.e. $\|A\|_{n}$ is an $n$-truncated type equipped with a map $|-|_{n}: A \rightarrow\|A\|_{n}$ such that for any $n$-truncated type $B$ the precomposition map $\left(\|A\|_{n} \rightarrow B\right) \rightarrow(A \rightarrow B)$ is an equivalence. A type $A$ is $n$-connected if $\|A\|_{n}$ is contractible. A function $f: A \rightarrow B$ is called $n$-truncated ( $n$-connected) if for all $y: B$ the type $\operatorname{fib}_{f}(y)$ is $n$-truncated ( $n$-connected). 
Properties of these notions are established in Chapter 7 of [The Univalent Foundations Program, Institute for Advanced Study 2013].

\section{Sequences and Sequential Colimits}

Our main ingredient, the sequential colimit, is a colimit of a diagram of a particular shape:

Definition 3.1. A sequence is a pair $(A, a)$ : Seq with

- $\mathrm{A}: \mathbb{N} \rightarrow \mathrm{U}$

- $\mathrm{a}: \Pi_{n: \mathbb{N}} \mathrm{A}(n) \rightarrow \mathrm{A}(n+1)$

Definition 3.2. The colimit $\operatorname{colim}(\mathrm{A}, \mathrm{a})$ of a sequence $(\mathrm{A}, \mathrm{a})$ is the higher inductive type generated by the following constructors:

$$
\begin{aligned}
& \iota: \Pi_{n: \mathbb{N}} \mathrm{A}(n) \rightarrow \operatorname{colim}(\mathrm{A}, \mathrm{a}) \\
& \kappa: \Pi_{n: \mathbb{N}} \Pi_{a: \mathrm{A}(n)} \iota(n+1, \mathrm{a}(n, a))=\iota(n, a)
\end{aligned}
$$

The constructor $\iota$ gives the canonical injection of $a: \mathrm{A}(n)$ into the colimit, whereas the constructor $\kappa$ provides the glue that ensures that the respective injections of the points $a: \mathrm{A}(n)$ and $\mathrm{a}(n, a): \mathrm{A}(n+1)$ into the colimit coincide. The higher dimensionality of this inductively-defined type comes from the fact that $\kappa$ does not construct a term of $\operatorname{colim}(A, a)$ itself but rather of the identity type over $\operatorname{colim}(A, a)$. We will sometimes denote $\operatorname{colim}(\mathrm{A}, \mathrm{a})$ by $\operatorname{colim}_{n}(\mathrm{~A}(n))$ or $\mathrm{A}_{\infty}$ if the maps can be inferred from the context.

The constructors in Definition 3.2 are accompanied by the following principle of induction: given

- $E: \operatorname{colim}(\mathrm{A}, \mathrm{a}) \rightarrow \mathrm{U}$

- $e: \Pi_{n: \mathbb{N}} \Pi_{a: \mathrm{A}(n)} E(\iota(n, a))$

- $p: \Pi_{n: \mathbb{N}} \Pi_{a: \mathrm{A}(n)} \kappa(n, a) \#_{E} e(n+1, \mathrm{a}(n, a))=e(n, a)$

we have a map ind $(E, e, p): \Pi_{x: \operatorname{colim}(\mathrm{A}, \mathrm{a})} E(x)$ such that for any $n: \mathbb{N}, a: \mathrm{A}(n)$,

- ind $(E, e, p) \iota(n, a) \equiv e(n, a)$

- $\operatorname{apd}_{\operatorname{ind}(E, e, p)}(\kappa(n, a))=p(n, a)$

The induction principle implies the following recursion principle: given

- $E: U$

- $e: \Pi_{n: \mathbb{N}} \mathrm{A}(n) \rightarrow E$

- $p: \Pi_{n: \mathbb{N}} \Pi_{a: \mathrm{A}(n)} e(n+1, \mathrm{a}(n, a))=e(n, a)$

there is a function $\operatorname{rec}(E, e, p): \operatorname{colim}(\mathrm{A}, \mathrm{a}) \rightarrow E$ such that for any $n: \mathbb{N}, a: \mathrm{A}(n)$,

- $\operatorname{rec}(E, e, p) \iota(n, a) \equiv e(n, a)$

- $\operatorname{ap}_{\operatorname{rec}(E, e, p)}(\kappa(n, a))=p(n, a)$

One important consequence of the induction principle is:

Lemma 3.3 (Uniqueness property of the sequential colimit). Two functions $F_{1}, F_{2}: \operatorname{colim}(\mathrm{A}, \mathrm{a}) \rightarrow$ E out of the sequential colimit are equal if there is a family of homotopies

- $\alpha: \Pi_{n: \mathbb{N}} F_{1} \circ \iota(n)=F_{2} \circ \iota(n)$

such that the diagram of identifications below commutes for all $n: \mathbb{N}, a: \mathrm{A}(n)$.

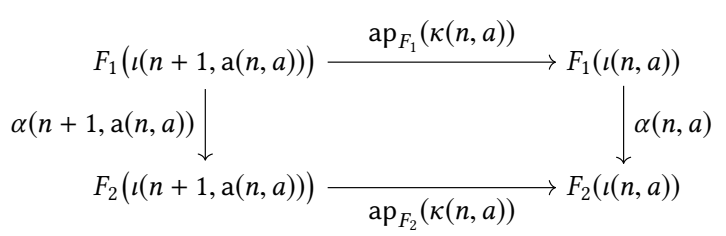

Morphisms of sequences are natural transformations:

Definition 3.4. Let $(A, a)$ and $\left(A^{\prime}, a^{\prime}\right)$ be sequences. A natural transformation $(\mathrm{A}, \mathrm{a}) \rightarrow\left(\mathrm{A}^{\prime}, \mathrm{a}^{\prime}\right)$ is a pair $(\tau, H)$ consisting of a family of maps

$$
\tau: \Pi_{n: \mathbb{N}} \mathrm{A}(n) \rightarrow \mathrm{A}^{\prime}(n)
$$

and a family of homotopies $H(n)$ witnessing that the diagram

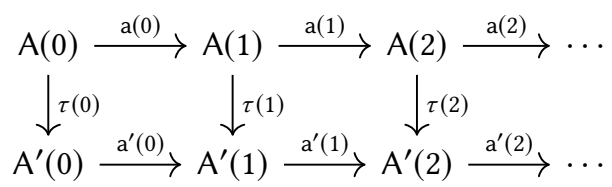

commutes, i.e. $H_{n}: \tau(n+1) \circ \mathrm{a}(n) \sim \mathrm{a}^{\prime}(n) \circ \tau(n)$.

A natural equivalence is a natural transformation $(\tau, H)$ where $\tau(n)$ is an equivalence for all $n$.

The identity and composition of natural transformations are defined in the obvious way.

Lemma 3.5 (Functoriality of the Sequential Colimit). We have the following:

1. A natural transformation $(\tau, H):(\mathrm{A}, \mathrm{a}) \rightarrow\left(\mathrm{A}^{\prime}, \mathrm{a}^{\prime}\right)$ induces a map colim $(\tau, H)$ or $\tau_{\infty}: \mathrm{A}_{\infty} \rightarrow \mathrm{A}_{\infty}^{\prime}$ given by

$$
\begin{aligned}
& \iota(n, a) \mapsto \iota(n, \tau(n, a)) \\
& \kappa(n, a) \mapsto \operatorname{ap}_{\iota(n+1)}(H(n, a)) \cdot \kappa(n, \tau(n, a))
\end{aligned}
$$

2. For $1:(\mathrm{A}, \mathrm{a}) \rightarrow(\mathrm{A}, \mathrm{a})$, we have $1_{\infty} \sim \mathrm{id}_{A_{\infty}}$.

3. Given $(\tau, H):(\mathrm{A}, \mathrm{a}) \rightarrow\left(\mathrm{A}^{\prime}, \mathrm{a}^{\prime}\right)$ and $(\sigma, K):\left(\mathrm{A}^{\prime}, \mathrm{a}^{\prime}\right) \rightarrow$ $\left(\mathrm{A}^{\prime \prime}, \mathrm{a}^{\prime \prime}\right)$, we have $(\sigma \circ \tau)_{\infty} \sim \sigma_{\infty} \circ \tau_{\infty}$.

4. Given $(\tau, H),\left(\tau^{\prime}, H^{\prime}\right):(\mathrm{A}, \mathrm{a}) \rightarrow\left(\mathrm{A}^{\prime}, \mathrm{a}^{\prime}\right)$, if there is a family of homotopies $q: \Pi_{n: \mathbb{N}} \tau(n) \sim \tau^{\prime}(n)$ such that the diagram of paths below commutes for all $n: \mathbb{N}, a: \mathrm{A}(n)$, then $\tau_{\infty} \sim \tau_{\infty}^{\prime}$.

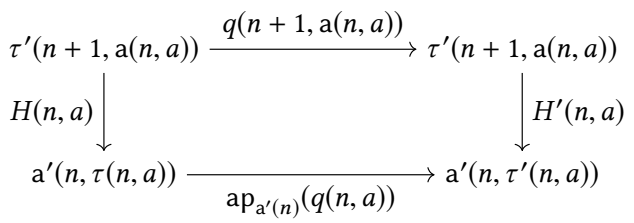

5. If $(\tau, H)$ is a natural equivalence then $\tau_{\infty}$ is an equivalence.

Given a sequence $(A, a)$, the colimit $\operatorname{colim}(A, a)$ is in fact equal to the colimit of the sequence

$$
\mathrm{A}(1) \stackrel{\mathrm{a}(1)}{\longrightarrow} \mathrm{A}(2) \stackrel{\mathrm{a}(2)}{\longrightarrow} \mathrm{A}(3) \stackrel{\mathrm{a}(3)}{\longrightarrow} \cdots
$$


which drops the first index. This is intuitively clear since any point $\iota(0, a)$ corresponding to $a: \mathrm{A}(0)$ is already present in the colimit as the point $\iota(1, \mathrm{a}(0, a))$ corresponding to $\mathrm{a}(0, a)$ : $A(1)$, up to typal equality. Formally:

Lemma 3.6. We have

$$
\operatorname{colim}(n \mapsto \mathrm{A}(n+1), n \mapsto \mathrm{a}(n+1))=\operatorname{colim}(\mathrm{A}, \mathrm{a})
$$

as witnessed by the equivalence

$$
\begin{aligned}
\iota(n, a) & \mapsto \iota(n+1, a) \\
\kappa(n, a) & \mapsto \kappa(n+1, a)
\end{aligned}
$$

Similarly:

Lemma 3.7. For $n: \mathbb{N}$ we have

$$
\operatorname{colim}(k \mapsto \mathrm{A}(n+k), k \mapsto \mathrm{a}(n+k))=\operatorname{colim}(\mathrm{A}, \mathrm{a})
$$

as witnessed by the equivalence

$$
\begin{aligned}
\iota(k, a) & \mapsto \iota(n+k, a) \\
\kappa(k, a) & \mapsto \kappa(n+k, a)
\end{aligned}
$$

A term $a: \mathrm{A}(n)$ can be lifted to the term $\mathrm{a}(n, a): \mathrm{A}(n+1)$, which can be lifted to the term $\mathrm{a}(n+1, \mathrm{a}(n, a)): \mathrm{A}(n+2)$, and so on. Formally, let $\Sigma \mathrm{A}:=\sum_{n: \mathbb{N}} \mathrm{A}(n)$ and define the liftings:

- $x^{+}: \Sigma \mathrm{A}$ for $x: \sum \mathrm{A}$ by $(n, a)^{+}:=(n+1, \mathrm{a}(n, a))$

- $x^{+k}: \sum \mathrm{A}$ for $x: \sum \mathrm{A}$ by $x^{+0}:=x, x^{+(k+1)}:=\left(x^{+k}\right)^{+}$

- $a^{+k}: \mathrm{A}(k)$ for $a: \mathrm{A}(0)$ by $a^{+0}:=a, a^{+(k+1)}:=\mathrm{a}\left(k, a^{+k}\right)$

Since any term $a: \mathrm{A}(n)$ can be seen as a term $a: \mathrm{A}^{\prime}(0)$ for the sequence $\left(\mathrm{A}^{\prime}, \mathrm{a}^{\prime}\right):=(k \mapsto \mathrm{A}(n+k), k \mapsto \mathrm{a}(n+k))$, the $k$-fold lifting in the latter sequence yields $a^{+k}: \mathrm{A}(n+k)$. The expected relationships hold: lifting is associative up to typal equality, i.e., we have $x^{++k}=x^{+k+1}$ for any $x: \Sigma \mathrm{A}$; for $a: \mathrm{A}(0)$ we have $(0, a)^{+k}=\left(k, a^{+k}\right)$; and for $a: \mathrm{A}(n)$ we have $(n, a)^{+k}=\left(n+k, a^{+k}\right)$.

\section{Fibered Sequences}

We now shift our attention to a dependent version of sequences:

Definition 4.1. A sequence fibered over a sequence $(A, a)$ is a pair $(B, b): \operatorname{FibSeq}(A, a)$ with

$$
\begin{aligned}
& \text { - } \mathrm{B}: \Sigma \mathrm{A} \rightarrow \mathrm{U} \\
& \text { - } \mathrm{b}: \Pi_{x: \Sigma \mathrm{A}} \mathrm{B}(x) \rightarrow \mathrm{B}\left(x^{+}\right)
\end{aligned}
$$

which can be visualized as the family of sequences below, one for each $x: \sum$ A.

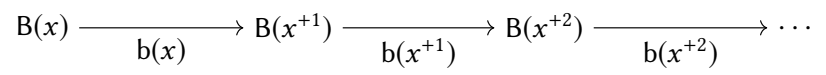

We say that $(\mathrm{B}, \mathrm{b})$ is equifibered if each $\mathrm{b}(x)$ is an equivalence.

The uniqueness property of the colimit plus univalence imply that type families over colim $(A, a)$ can be characterized as equifibered sequences over $(A, a)$ :
Lemma 4.2. The type $\operatorname{colim}(\mathrm{A}, \mathrm{a}) \rightarrow \mathrm{U}$ is equivalent to the type of equifibered sequences over $(\mathrm{A}, \mathrm{a})$, i.e.

$$
\begin{aligned}
& \operatorname{colim}(\mathrm{A}, \mathrm{a}) \rightarrow \mathrm{U} \\
\simeq & \sum_{\mathrm{C}: \Pi_{n: \mathbb{N}} \mathrm{A}(n) \rightarrow \mathrm{U}} \Pi_{n: \mathbb{N}} \Pi_{a: \mathrm{A}(n)} \mathrm{C}(n+1, \mathrm{a}(n, a)) \simeq \mathrm{C}(n, a)
\end{aligned}
$$

Since for any $n: \mathbb{N}$ we can form the type $\sum_{a: \mathrm{A}(n)} \mathrm{B}(n, a)$, there is a natural way to combine $(A, a)$ and $(B, b)$ into a sequence:

Definition 4.3. We define the sequence $\Sigma_{(A, a)}(B, b)$ as the pair

$$
\begin{aligned}
& \text { - } n \mapsto \sum_{a: \mathrm{A}(n)} \mathrm{B}(n, a) \\
& \text { - } n,(a, b) \mapsto(\mathrm{a}(n, a), \mathrm{b}((n, a), b))
\end{aligned}
$$

The canonical projection $\operatorname{colim}\left(\Sigma_{(\mathrm{A}, \mathrm{a})}(\mathrm{B}, \mathrm{b})\right) \rightarrow \operatorname{colim}(\mathrm{A}, \mathrm{a})$ is defined by:

$$
\begin{aligned}
\iota(n,(a, b)) & \mapsto \iota(n, a) \\
\kappa(n,(a, b)) & \mapsto \kappa(n, a)
\end{aligned}
$$

We can informally describe the type $\operatorname{colim}\left(\Sigma_{(\mathrm{A}, \mathrm{a})}(\mathrm{B}, \mathrm{b})\right)$ as a "colim after $\Sigma$ ". The natural questions now are, what does the dual type " $\Sigma$ after colim" look like, and are the two types equal? We will answer the former in this section and the latter in the rest of the paper.

To define a dependent sum of colimits, we need a type family $B_{\infty}: A_{\infty} \rightarrow U$. By Lemma 4.2 it suffices to construct an equifibered family $\left(\mathrm{C}_{\infty}, \mathrm{c}_{\infty}\right)$, where $\mathrm{C}_{\infty}: \Pi_{n: \mathbb{N}} \mathrm{A}(n) \rightarrow \mathrm{U}$ and $\mathrm{c}_{\infty}: \Pi_{n: \mathbb{N}} \Pi_{a: \mathrm{A}(n)} \mathrm{C}_{\infty}(n+1, \mathrm{a}(n, a)) \simeq \mathrm{C}_{\infty}(n, a)$. For the former, we define $\mathrm{C}: \Pi_{n: \mathbb{N}} \mathrm{A}(n) \rightarrow \mathbb{N} \rightarrow \mathrm{U}$ by

$$
\begin{aligned}
& \mathrm{C}(n, a, 0):=\mathrm{B}(n, a) \\
& \mathrm{C}(n, a, k+1):=\mathrm{C}(n+1, \mathrm{a}(n, a), k)
\end{aligned}
$$

and c $: \Pi_{n: \mathbb{N}} \Pi_{a: \mathrm{A}(n)} \Pi_{k: \mathbb{N}} \mathrm{C}(n, a, k) \rightarrow \mathrm{C}(n, a, k+1)$ by

$$
\begin{aligned}
& \mathrm{c}(n, a, 0):=\mathrm{b}(n, a) \\
& \mathrm{c}(n, a, k+1):=\mathrm{c}(n+1, \mathrm{a}(n, a), k)
\end{aligned}
$$

and put

$$
\mathrm{C}_{\infty}(n, a):=\operatorname{colim}(k \mapsto \mathrm{C}(n, a, k), k \mapsto \mathrm{c}(n, a, k))
$$

To define $\mathrm{c}_{\infty}(x)$, we appeal to Lemma 3.6 to obtain the equivalence:

$$
\begin{aligned}
& \mathrm{C}_{\infty}(n+1, \mathrm{a}(n, a)) \\
\equiv & \operatorname{colim}(k \mapsto \mathrm{C}(n+1, \mathrm{a}(n, a), k), k \mapsto \mathrm{c}(n+1, \mathrm{a}(n, a), k)) \\
\simeq & \operatorname{colim}(k \mapsto \mathrm{C}(n, a, k), k \mapsto \mathrm{c}(n, a, k)) \\
\equiv & \mathrm{C}_{\infty}(n, a)
\end{aligned}
$$

We have thus constructed $\mathrm{B}_{\infty}: \mathrm{A}_{\infty} \rightarrow \mathrm{U}$, where $\mathrm{B}_{\infty}(l(n, a)) \equiv$ $\mathrm{C}_{\infty}(n, a)$ and we have a witness $\beta_{\mathrm{B}_{\infty}}(n, a): \overline{\operatorname{ap}_{\mathrm{B}_{\infty}}(\kappa(n, a))}=$ $\mathrm{c}(n, a)$ (we recall that overline denotes a map induced by a path between types). 


\section{Colimits and Sums}

To show colim $\left(\sum_{(\mathrm{A}, \mathrm{a})}(\mathrm{B}, \mathrm{b})\right) \simeq \sum_{x: \mathrm{A}_{\infty}} \mathrm{B}_{\infty}(x)$, we want to construct mutually inverse functions between these types. From left to right, the only reasonable action on points is to map $\iota(n,(a, b))$ to the pair $(\iota(n, a), \iota(0, b))$, which makes sense since $b: \mathrm{B}(n, a)$. Mapping the path constructor $\kappa(n,(a, b))$ requires a path

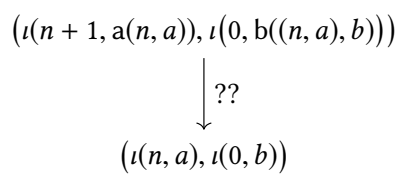

The constructor $\kappa(0, b)$ for the sequential colimit $\mathrm{C}_{\infty}(n, a)$ gives us a path $\iota(1, \mathrm{~b}((n, a), b))=\iota(0, b)$ that we can use as follows:

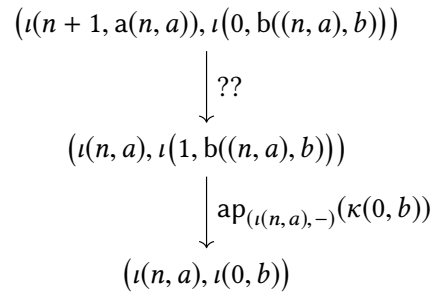

In the remaining path, the constructor $\kappa(n, a)$ for the sequential colimit $\mathrm{A}_{\infty}$ gives us equality of the first components, and hence a map $\overline{\operatorname{ap}_{\mathrm{B}_{\infty}}(\kappa(n, a))}: \mathrm{C}_{\infty}(n+1, \mathrm{a}(n, a)) \rightarrow$ $\mathrm{C}_{\infty}(n, a)$. This function is equal to $\mathrm{c}_{\infty}(n, a)$, as witnessed by $\beta_{\mathrm{B}_{\infty}}(n, a)$, and the latter map carries $\iota(0, \mathrm{~b}((n, a), b))$ precisely to $\iota(1, \mathrm{~b}((n, a), b))$. We can generalize this situation as follows: given

$$
\begin{aligned}
& \text { - } x_{1}, x_{2}: \mathrm{A}_{\infty} \text { and } \alpha: x_{1}=x_{2} \\
& \text { - } F: \mathrm{B}_{\infty}\left(x_{1}\right) \rightarrow \mathrm{B}_{\infty}\left(x_{2}\right) \\
& \text { - } F_{\star}: \overline{\operatorname{ap}_{\mathrm{B}_{\infty}}(\alpha)}=F \\
& \text { - } y: \mathrm{B}_{\infty}\left(x_{1}\right)
\end{aligned}
$$

we have a path $\Delta\left(\alpha, F_{\star}, y\right):\left(x_{1}, y\right)=\left(x_{2}, F(y)\right)$ defined by induction on $\alpha$ and $F_{\star}$. This completes the definition of a function from left to right, and allows us to concisely state our main result:

Theorem 5.1 (Interaction between colim and $\Sigma$ ). We have:

$$
\operatorname{colim}\left(\sum_{(\mathrm{A}, \mathrm{a})}(\mathrm{B}, \mathrm{b})\right)=\Sigma_{x: \mathrm{A}_{\infty}} \mathrm{B}_{\infty}(x)
$$

as witnessed by the equivalence $\mathrm{F}$ below:

$$
\begin{aligned}
\iota(n,(a, b)) \mapsto & (\iota(n, a), \iota(0, b)) \\
\kappa(n,(a, b)) \mapsto & \Delta\left(\kappa(n, a), \beta_{\mathrm{B}_{\infty}}(n, a), \iota(0, \mathrm{~b}((n, a), b))\right) . \\
& \operatorname{ap}_{(\iota(n, a),-)}(\kappa(0, b))
\end{aligned}
$$

that commutes with the canonical projections:

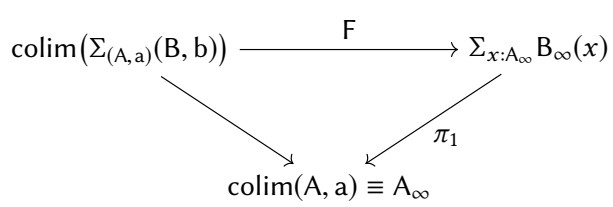

with the one on the left as given in Definition 4.3.

Proof of commutativity. We use the uniqueness property of the colimit. On points $\iota(n,(a, b))$ the maps agree definitionally. To show that $\kappa(n, a)=\operatorname{ap}_{\pi_{1}}\left(\operatorname{ap}_{\mathrm{F}}(\kappa(n,(a, b)))\right)$, we use the following easy generalization: given

- $x_{1}, x_{2}: \mathrm{A}_{\infty}$ and $\alpha: x_{1}=x_{2}$

- $y_{1}: \mathrm{B}_{\infty}\left(x_{1}\right)$ and $y_{2}: \mathrm{B}_{\infty}\left(x_{2}\right)$

- $F: \mathrm{B}_{\infty}\left(x_{1}\right) \rightarrow \mathrm{B}_{\infty}\left(x_{2}\right)$

- $F_{\star}: \overline{\operatorname{ap}_{\mathrm{B}_{\infty}}(\alpha)}=F$

- $\beta: F\left(y_{1}\right)=y_{2}$

we have $\alpha=\operatorname{ap}_{\pi_{1}}\left(\Delta\left(\alpha, F_{\star}, y_{1}\right) \cdot \operatorname{ap}_{\left(x_{2},-\right)}(\beta)\right)$.

Now it "only" remains to show that $F$ is indeed an equivalence, by exhibiting an inverse. However, induction on the sum of sequential colimits is significantly harder than induction on the sequential colimit of the sum: the former requires two nested colimit inductions and results not in a point case and a path case, as before, but rather in point-point, point-path, path-point, path-path cases.

Worse yet, it appears we may have to carry out this form of induction twice: once when constructing the inverse to $\mathrm{F}$ and again when proving that the two maps compose to the identity on $\Sigma_{x: \mathrm{A}_{\infty}} \mathrm{B}_{\infty}(x)$. Or do we? If the sum of colimits is to be equal to the colimit of the sum, then the two types better have the same induction principle! In other words, we should be able to construct a map out of $\Sigma_{x: \mathrm{A}_{\infty}} \mathrm{B}_{\infty}(x)$ as if we were constructing a map out of $\operatorname{colim}\left(\Sigma_{(\mathrm{A}, \mathrm{a})}(\mathrm{B}, \mathrm{b})\right)$, using $\mathrm{F}$ to appropriately mediate between the two types:

Lemma 5.2 (Induction on the sum of sequential colimits). Given

$$
\begin{aligned}
\text { - } \mathrm{E}: & \left(\sum_{x: \mathrm{A}_{\infty}} \mathrm{B}_{\infty}(x)\right) \rightarrow \mathrm{U} \\
\text { - } \mathrm{e}: & \Pi_{n: \mathbb{N}} \Pi_{a: \mathrm{A}(n)} \Pi_{b: \mathrm{B}(n, a)} \mathrm{E}(\mathrm{F}(\iota(n,(a, b)))) \\
\text { - } \mathrm{p}: & \Pi_{n: \mathbb{N}} \Pi_{a: \mathrm{A}(n)} \Pi_{b: \mathrm{B}(n, a)} \mathrm{ap}_{\mathrm{F}}(\kappa(n,(a, b))) \#_{\mathrm{E}} \\
& \mathrm{e}(n+1, \mathrm{a}(n, a), \mathrm{b}((n, a), b))=\mathrm{e}(n, a, b)
\end{aligned}
$$

there is a function $\mathrm{G}: \Pi_{y:\left(\Sigma_{x: \mathrm{A}_{\infty}} \mathrm{B}_{\infty}(x)\right)} \mathrm{E}(y)$ such that for any $n: \mathbb{N}, a: \mathrm{A}(n), b: \mathrm{B}(n, a)$,

- $\mathrm{G}(\mathrm{F}(\iota(n,(a, b)))) \equiv \mathrm{e}(n, a, b)$

- $\operatorname{apd}_{\mathrm{G}}\left(\operatorname{ap}_{\mathrm{F}}(\kappa(n,(a, b)))\right)=\mathrm{p}(n, a, b)$

Of course, to establish this lemma we still need to carry out the more difficult nested induction - but only once, and then our work is essentially done. Lemma 5.2 easily implies the corresponding recursion principle:

Lemma 5.3 (Recursion on the sum of sequential colimits). Given

$$
\begin{aligned}
\text { - } & E: \mathrm{U} \\
\text { - } & e: \Pi_{n: \mathbb{N}} \Pi_{a: \mathrm{A}(n)} \mathrm{B}(n, a) \rightarrow \mathrm{E} \\
\text { - } p: & \Pi_{n: \mathbb{N}} \Pi_{a: \mathrm{A}(n)} \Pi_{b: \mathrm{B}(n, a)} \\
& e(n+1, \mathrm{a}(n, a), \mathrm{b}((n, a), b))=e(n, a, b)
\end{aligned}
$$


there is a function $G:\left(\sum_{x: \mathrm{A}_{\infty}} \mathrm{B}_{\infty}(x)\right) \rightarrow$ E such that for any $n: \mathbb{N}, a: \mathrm{A}(n), b: \mathrm{B}(n, a)$,

- $G(\mathrm{~F}(\iota(n,(a, b)))) \equiv e(n, a, b)$

- $\operatorname{ap}_{G}\left(\operatorname{ap}_{\mathrm{F}}(\kappa(n,(a, b)))\right)=p(n, a, b)$

Lemma 5.2 now easily implies the following:

Lemma 5.4 (Uniqueness property of the sum of sequential colimits). Two maps $G_{1}, G_{2}:\left(\sum_{x: \mathrm{A}_{\infty}} \mathrm{B}_{\infty}(x)\right) \rightarrow E$ out of the sum of colimits are equal if the maps $G_{1} \circ \mathrm{F}$ and $G_{2} \circ \mathrm{F}$ are, i.e. $\mathrm{F}$ is epic.

The proof of our main result, Theorem 5.1, is now easy:

Proof of equivalence. We construct the inverse $\mathrm{F}^{-1}$ by recursion on $\Sigma_{x: \mathrm{A}_{\infty}} \mathrm{B}_{\infty}(x)$ as in Lemma 5.3:

$$
\begin{aligned}
\mathrm{F}(\iota(n,(a, b))) & \mapsto \iota(n,(a, b)) \\
\operatorname{ap}_{\mathrm{F}}(\kappa(n,(a, b))) & \mapsto \kappa(n,(a, b))
\end{aligned}
$$

The equality $\mathrm{F}^{-1} \circ \mathrm{F}=$ id is now immediate from the uniqueness property of the sequential colimit and the equality $F \circ F^{-1}=$ id from the uniqueness property of the sum of sequential colimits.

In the next section we give the proof of Lemma 5.2.

\section{Induction on the Sum of Sequential Colimits}

To prove Lemma 5.2, we want to construct the curried version of $\mathrm{G}$, the map $x, y \mapsto \mathrm{G}(x, y): \Pi_{x: \mathrm{A}_{\infty}} \Pi_{y: \mathrm{B}_{\infty}(x)} \mathrm{E}(x, y)$, by nested sequential colimit induction. For the outer induction on $\mathrm{A}_{\infty}$, we need to map $\iota(n, a)$ to a function

- $\mathrm{g}(n, a): \Pi_{y: \mathrm{C}_{\infty}(n, a)} \mathrm{E}(\iota(n, a), y)$

and $\kappa(n, a)$ to an equality

$$
\kappa(n, a) \#_{\left(x \mapsto \Pi_{y: \mathrm{B}_{\infty}(x)} \mathrm{E}(x, y)\right)} \mathrm{g}(n+1, \mathrm{a}(n, a))=\mathrm{g}(n, a)
$$

We want to define $\mathrm{g}(n, a)$ by induction on the sequential colimit $\mathrm{C}_{\infty}(n, a)$. We thus need $\mathrm{h}$ and $\mathrm{H}$ such that for any $k: \mathbb{N}$ and $b: \mathrm{C}(n, a, k)$,

- $\mathrm{h}(n, a, k, b): \mathrm{E}(\iota(n, a), \iota(k, b))$

- $\mathrm{H}(n, a, k, b): \kappa(k, b) \#_{\mathrm{E}(\iota(n, a),-)}$ $\mathrm{h}(n, a, k+1, \mathrm{c}(n, a, k, b))=\mathrm{h}(n, a, k, b)$

The obvious way to proceed now would be to begin defining $\mathrm{h}$ and $\mathrm{H}$ right away and establish Equality (1) post-hoc. This may result in more work than necessary though: perhaps if we examine this equality first we can get some insight into how to best define $\mathrm{h}$ and $\mathrm{H}$ so that only a (relatively) small amount of effort is needed for the path-point and pathpath cases.

We start by reformulating Equality (1) in a way that makes it easier to understand. The maps $\mathrm{g}(n+1, \mathrm{a}(n, a))$ and $\mathrm{g}(n, a)$ have different domains: the former takes arguments from the sequential colimit $\mathrm{C}_{\infty}(n+1, \mathrm{a}(n, a))$ and the latter from $\mathrm{C}_{\infty}(n, a)$. But these colimits are equivalent via $\mathrm{c}_{\infty}(n, a)$, so relating the maps $\mathrm{g}(n+1, \mathrm{a}(n, a))$ and $\mathrm{g}(n, a)$ comes down to relating $\mathrm{g}(n+1, \mathrm{a}(n, a), y)$ and $\mathrm{g}\left(n, a, \mathrm{c}_{\infty}(n, a, y)\right)$ for any argument $y: \mathrm{C}_{\infty}(n+1, \mathrm{a}(n, a))$. The respective values lie in different fibers of $\mathrm{E}$, namely over $(\iota(n+1, \mathrm{a}(n, a)), y)$ versus over $\left(\iota(n, a), \mathrm{c}_{\infty}(n, a, y)\right)$, but the path $\Delta\left(\kappa(n, a), \beta_{\mathrm{B}_{\infty}}(n, a), y\right)$ equates the pairs.

Thus, we have the following more verbose but easier to use reformulation:

$$
\begin{aligned}
& \Pi_{y: \mathrm{C}_{\infty}(n+1, \mathrm{a}(n, a))} \mathrm{g}\left(n, a, \mathrm{c}_{\infty}(n, a, y)\right)= \\
& \quad \Delta\left(\kappa(n, a), \beta_{\mathrm{B}_{\infty}}(n, a), y\right){ }_{E} \mathrm{~g}(n+1, \mathrm{a}(n, a), y)
\end{aligned}
$$

Of course, we must show this is equivalent to the original formulation. We do so via a generalization: given

- $x_{1}, x_{2}: \mathrm{A}_{\infty}$ and $\alpha: x_{1}=x_{2}$

- $F: \mathrm{B}_{\infty}\left(x_{1}\right) \rightarrow \mathrm{B}_{\infty}\left(x_{2}\right)$

- $F_{\star}: \overline{\operatorname{ap}_{\mathrm{B}_{\infty}}(\alpha)}=F$

- $f_{1}: \Pi_{y: \mathrm{B}_{\infty}\left(x_{1}\right)} \mathrm{E}\left(x_{1}, y\right)$

- $f_{2}: \Pi_{y: B_{\infty}\left(x_{2}\right)} \mathrm{E}\left(x_{2}, y\right)$

we have an equivalence $\mathrm{I}\left(\alpha, \mathrm{F}_{\star}, f_{1}, f_{2}\right)$ :

$$
\begin{gathered}
\alpha \#_{\left(x \mapsto \Pi_{y: \mathrm{B}_{\infty}(x)} E(x, y)\right)} f_{1}=f_{2} \\
\simeq \\
\Pi_{y: \mathrm{B}_{\infty}\left(x_{1}\right)} f_{2}(F(y))=\Delta\left(\alpha, F_{\star}, y\right) \#_{E} f_{1}(y)
\end{gathered}
$$

We define I by induction on $\alpha$ and $F_{\star}$. This reduces the above equivalence to $\left(f_{1}=f_{2}\right) \simeq\left(f_{2} \sim f_{1}\right)$, witnessed by the symmetry $\left(f_{1}=f_{2}\right) \simeq\left(f_{2}=f_{1}\right)$ and the map $\operatorname{hap}\left(f_{2}, f_{1}\right)$.

It thus suffices to produce a witness $\omega(n, a)$ for (2), which will give us the desired witness for (1), explicitly described below:

$$
\mathrm{I}\left(\kappa(n, a), \beta_{\mathrm{B}_{\infty}}(n, a), \mathrm{g}(n+1, \mathrm{a}(n, a)), \mathrm{g}(n, a)\right)^{-1} \omega(n, a)
$$

We construct $\omega(n, a)$ by induction on the sequential colimit $\mathrm{C}_{\infty}(n+1, \mathrm{a}(n, a))$. So for any $k: \mathbb{N}$ and $b: \mathrm{C}(n, a, k+1)$ we need

- $\mu(n, a, k, b): \mathrm{g}\left(n, a, \mathrm{c}_{\infty}(n, a, \iota(k, b))\right)=$ $\Delta\left(\kappa(n, a), \beta_{\mathrm{B}_{\infty}}(n, a), \iota(k, b)\right) \#_{E} \mathrm{~g}(n+1, \mathrm{a}(n, a), \iota(k, b))$

and an equality:

$$
\begin{aligned}
& \kappa(k, b) \#_{y \mapsto \mathrm{g}}\left(n, a, \mathrm{c}_{\infty}(n, a, y)\right)=\Delta\left(\kappa(n, a), \beta_{\mathrm{B}_{\infty}}(n, a), y\right) \#_{E} \mathrm{~g}(n+1, \mathrm{a}(n, a), y) \\
& \mu(n, a, k+1, \mathrm{c}(n, a, k+1, b))=\mu(n, a, k, b)
\end{aligned}
$$

We can now divide the proof into the following steps:

- The point-point case: defining h.

- The path-point case: defining $\mu$.

- The point-path case: defining $\mathrm{H}$.

- The path-path case: showing that (3) holds.

- Establishing the computation rules. 


\subsection{The point-point and path-point cases}

We first simplify the type of $\mu$. By definition of $g$ we have

$$
\mathrm{g}(n+1, \mathrm{a}(n, a), \iota(k, b)) \equiv \mathrm{h}(n+1, \mathrm{a}(n, a), k, b)
$$

and similarly

$$
\begin{aligned}
\mathrm{g}\left(n, a, \mathrm{c}_{\infty}(n, a, \iota(k, b))\right) & \equiv \mathrm{g}(n, a, \iota(k+1, b)) \\
& \equiv \mathrm{h}(n, a, k+1, b)
\end{aligned}
$$

hence we need

$$
\begin{aligned}
& \text { - } \mu(n, a, k, b): \mathrm{h}(n, a, k+1, b)= \\
& \Delta\left(\kappa(n, a), \beta_{\mathrm{B}_{\infty}}(n, a), \iota(k, b)\right) \#_{E} \mathrm{~h}(n+1, \mathrm{a}(n, a), k, b)
\end{aligned}
$$

The type of $\mu$ thus shows how to express $\mathrm{h}(\cdot, \cdot, k+1, \cdot)$ in terms of $\mathrm{h}(\cdot, \cdot, k, \cdot)$, giving us an inductive definition of $\mathrm{h}$ :

$$
\begin{aligned}
\mathrm{h}(n, a, k+1, b) & := \\
\Delta\left(\kappa(n, a), \beta_{\mathrm{B}_{\infty}}(n, a), \iota(k, b)\right){ }^{\#_{E}} \mathrm{~h}(n+1, \mathrm{a}(n, a), k, b) &
\end{aligned}
$$

In the zero case, we need a point in the fiber $\mathrm{E}(\iota(n, a), \iota(0, b))$ and this is just the assumption $\mathrm{e}(n, a, b): \mathrm{E}(\mathrm{F}(\iota(n,(a, b))))$, so

$$
\mathrm{h}(n, a, 0, b):=\mathrm{e}(n, a, b)
$$

We let $\mu(n, a, k, b)$ be reflexivity.

\subsection{The point-path and path-path cases}

As in the previous subsection, we try to show that (3) holds before defining $\mathrm{H}$, gaining an insight into what $\mathrm{H}$ should look like. We again start by replacing (3) with something equivalent but easier to understand. To figure out what the transport does, we would like to appeal to path induction. So the first step is to replace the path $\kappa(k, b)$ together with its endpoints $\iota(k+1, \mathrm{c}(n, a, k+1, b))$ and $\iota(k, b)$ by something more general:

$$
\text { - } y_{1}, y_{2}: \mathrm{C}_{\infty}(n+1, \mathrm{a}(n, a)) \text { and } \beta: y_{1}=y_{2}
$$

We replace $\mu(n, a, k+1, \mathrm{c}(n, a, k+1, b))$ and $\mu(n, a, k, b)$ (which are just reflexivities) by arbitrary paths

$$
\begin{array}{ll}
\text { - } & u_{1}: \mathrm{g}\left(n, a, \mathrm{c}_{\infty}\left(n, a, y_{1}\right)\right)= \\
& \Delta\left(\kappa(n, a), \beta_{\mathrm{B}_{\infty}}(n, a), y_{1}\right) \#_{\mathrm{E}} \mathrm{g}\left(n+1, \mathrm{a}(n, a), y_{1}\right) \\
\text { - } & u_{2}: \mathrm{g}\left(n, a, \mathrm{c}_{\infty}\left(n, a, y_{2}\right)\right)= \\
& \Delta\left(\kappa(n, a), \beta_{\mathrm{B}_{\infty}}(n, a), y_{2}\right) \#_{\mathrm{E}} \mathrm{g}\left(n+1, \mathrm{a}(n, a), y_{2}\right)
\end{array}
$$

But the path $\kappa(k, b)$ was not arbitrary at all: we had the further piece of information that

$$
\operatorname{ap}_{\mathrm{c}_{\infty}(n, a)}(\kappa(k, b))=\kappa(k+1, b)
$$

This is just the second computation rule of $\mathrm{c}_{\infty}(n, a)$, the witness for which we call $\beta_{\mathrm{c}_{\infty}}(n, a, k, b)$. We capture this abstractly as having:

- $\gamma: \mathrm{c}_{\infty}\left(n, a, y_{1}\right)=\mathrm{c}_{\infty}\left(n, a, y_{2}\right)$

- $\theta: \operatorname{ap}_{\mathrm{c}_{\infty}(n, a)}(\beta)=\gamma$
In this abstract scenario, we want to relate $u_{1}$ and $u_{2}$ over the path $\beta$. Since $u_{1}$ is a path in $\mathrm{E}\left(\iota(n, a), \mathrm{c}\left(n, a, y_{1}\right)\right)$ and $u_{2}$ is a path in $\mathrm{E}\left(\iota(n, a), \mathrm{c}\left(n, a, y_{2}\right)\right)$, to relate them we first need to have them in the same fiber of $E$. The two fibers of $E$ can be related by the transport of the type family $\mathrm{E}(\iota(n, a),-)$ over $\operatorname{ap}_{\mathrm{c}_{\infty}(n, a)}(\beta)$, which we could use to carry $u_{1}$ over. But since we know $\operatorname{ap}_{\mathrm{c}_{\infty}(n, a)}(\beta)=\gamma$, we can use the transport over $\gamma$ directly. So we want to fill the diagram in Figure 1. Using dependent application of $g$, we can fill edges as shown in Figure 2 . The remaining edge can be seen as witnessing the naturality of $\Delta\left(\kappa(n, a), \beta_{\mathrm{B}_{\infty}}(n, a)\right)$. To construct it, we define a homotopy

$$
\text { - } \varepsilon(\beta, \gamma, \theta): \gamma \#_{\mathrm{E}(\iota(n, a),-)} \Delta\left(\kappa(n, a), \beta_{\mathrm{B}_{\infty}}(n, a), y_{1}\right) \#_{\mathrm{E}} \sim
$$$$
\Delta\left(\kappa(n, a), \beta_{\mathrm{B}_{\infty}}(n, a), y_{2}\right) \#_{\mathrm{E}} \beta \#_{\mathrm{E}(\iota(n+1, \mathrm{a}(n, a)),-)}
$$

by induction on $\beta$ and $\theta$. We can thus complete the diagram as in Figure 3.

Putting all this together, we have the following generalization: given:

- $y_{1}, y_{2}: \mathrm{C}_{\infty}(n+1, \mathrm{a}(n, a))$ and $\beta: y_{1}=y_{2}$

- $\gamma: \mathrm{c}_{\infty}\left(n, a, y_{1}\right)=\mathrm{c}_{\infty}\left(n, a, y_{2}\right)$

- $\theta: \operatorname{ap}_{\mathrm{c}_{\infty}(n, a)}(\beta)=\gamma$

- $u_{1}: \mathrm{g}\left(n, a, \mathrm{c}_{\infty}\left(n, a, y_{1}\right)\right)=$ $\Delta\left(\kappa(n, a), \beta_{\mathrm{B}_{\infty}}(n, a), y_{1}\right) \#_{\mathrm{E}} \mathrm{g}\left(n+1, \mathrm{a}(n, a), y_{1}\right)$

- $u_{2}: \mathrm{g}\left(n, a, \mathrm{c}_{\infty}\left(n, a, y_{2}\right)\right)=$ $\Delta\left(\kappa(n, a), \beta_{\mathrm{B}_{\infty}}(n, a), y_{2}\right) \#_{\mathrm{E}} \mathrm{g}\left(n+1, \mathrm{a}(n, a), y_{2}\right)$

we have the equivalence in Figure 4, asserting that the commutativity of the diagram in Figure 3 is equivalent to $u_{1}$ and $u_{2}$ being suitably related over $\beta$. To show this, we perform induction on $\beta$ and $\theta$. This reduces the goal to showing that $u_{1}=u_{2}$ is equivalent to $\operatorname{refl} \cdot u_{2}=\operatorname{ap}_{\mathrm{id}}\left(u_{1}\right) \cdot \operatorname{refl} \cdot \operatorname{refl}$ and this is clearly the case.

Our intended instantiation is thus:

- $y_{1}:=\iota(k+1, \mathrm{c}(n, a, k+1, b))$

- $y_{2}:=\imath(k, b)$

- $\beta:=\kappa(k, b)$

- $\gamma:=\kappa(k+1, b)$

- $\theta:=\beta_{\mathrm{c}_{\infty}}(n, a, k, b)$

- $u_{1}:=\mu(n, a, k+1, \mathrm{c}(n, a, k+1, b)) \equiv \operatorname{refl}$

- $u_{2}:=\mu(n, a, k, b) \equiv \mathrm{refl}$

So to prove (3) it suffices to establish the equality in Figure 5. On the left hand side, the path $\operatorname{apd}_{\mathrm{g}(n, a)}(\kappa(k+1, b))$ is supposed to be equal to $\mathrm{H}(n, a, k+1, b)$; on the right hand side, the path apd $\mathrm{g}_{\mathrm{g}(n+1, \mathrm{a}(n, a))}(\kappa(k, b))$ is supposed to be equal to $\mathrm{H}(n+1, \mathrm{a}(n, a), k, b)$. On the right hand side, the point $\mathrm{g}(n+1, \mathrm{a}(n, a), \iota(k+1, \mathrm{c}(n, a, k+1, b)))$ is supposed to be definitionally equal to $\mathrm{h}(n+1, \mathrm{a}(n, a), k+1, \mathrm{c}(n, a, k+1, b))$. Thus, we have expressed $\mathrm{H}(\cdot, \cdot, k+1, \cdot)$ in terms of $\mathrm{H}(\cdot, \cdot, k, \cdot)$, which gives us the successor step of the definition of $\mathrm{H}$ by induction on $k$ (see Figure 6). 


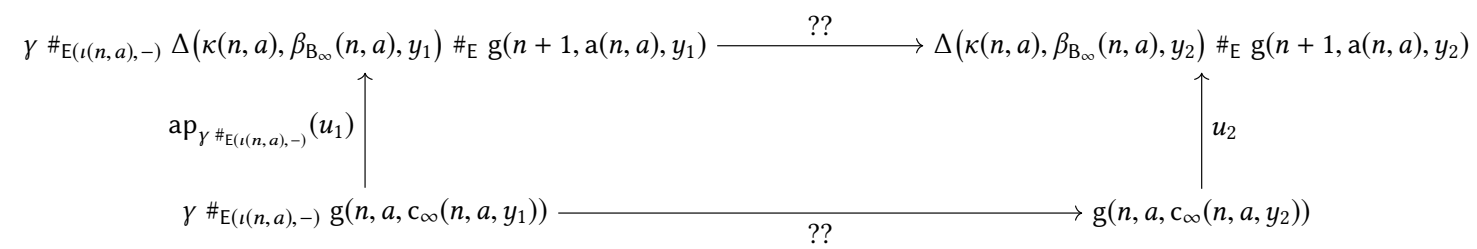

Figure 1. Filling the diagram

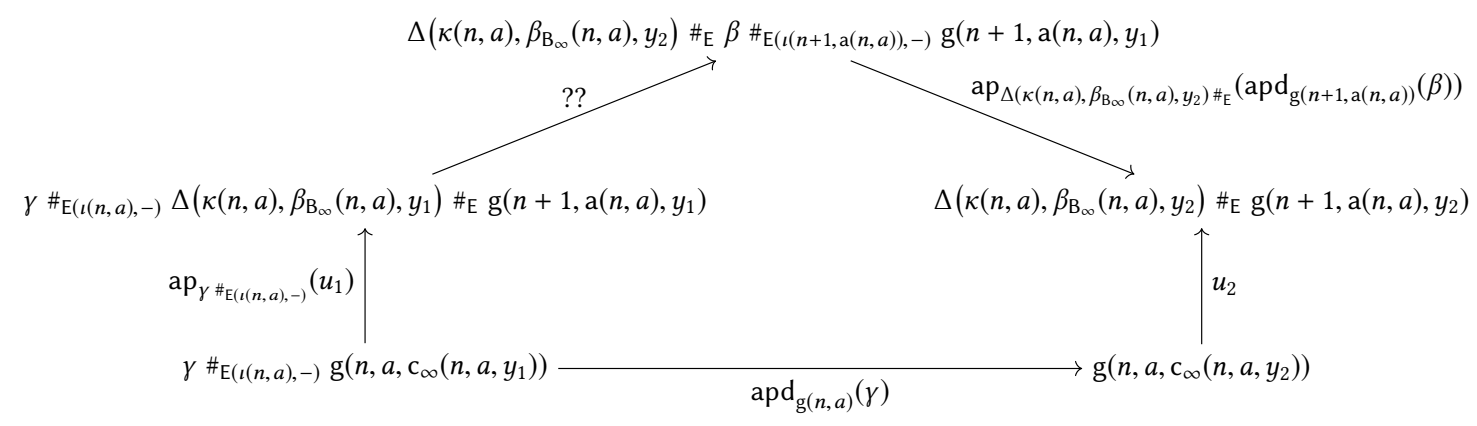

Figure 2. Filling the diagram, continued

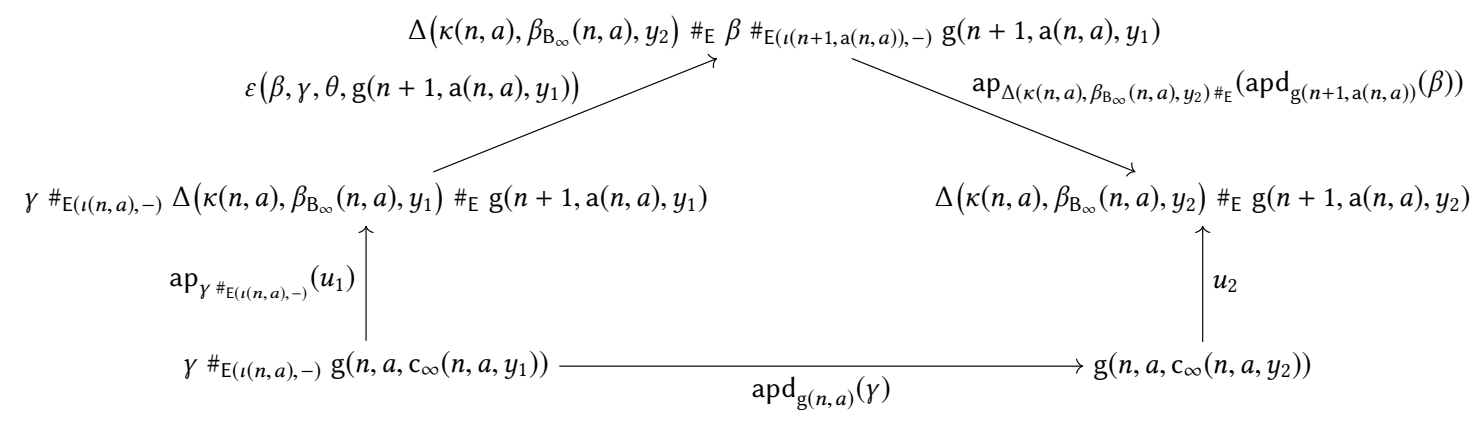

Figure 3. Filling the diagram, continued

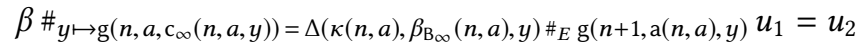

$$
\begin{aligned}
& \simeq \\
& \operatorname{apd}_{\mathrm{g}(n, a)}(\gamma) \cdot u_{2}=\operatorname{ap}_{\gamma \#_{\mathrm{E}(t(n, a),-)}}\left(u_{1}\right) \cdot \varepsilon\left(\beta, \gamma, \theta, \mathrm{g}\left(n+1, \mathrm{a}(n, a), y_{1}\right)\right) \cdot \\
& \operatorname{ap}_{\Delta\left(\kappa(n, a), \beta_{\mathrm{B}_{\infty}}(n, a), y_{2}\right) \#_{\mathrm{E}}}\left(\operatorname{apd}_{\mathrm{g}(n+1, \mathrm{a}(n, a))}(\beta)\right)
\end{aligned}
$$

Figure 4. Equality of $u_{1}$ and $u_{2}$ over $\beta$ is the same as the commutativity of the diagram in Figure 3

$$
\begin{aligned}
\operatorname{apd}_{\mathrm{g}(n, a)}(\kappa(k+1, b))= \\
\quad \varepsilon\left(\kappa(k, b), \kappa(k+1, b), \beta_{\mathrm{c}_{\infty}}(n, a, k, b), \mathrm{g}(n+1, \mathrm{a}(n, a), \iota(k+1, \mathrm{c}(n, a, k+1, b)))\right) . \\
\quad \operatorname{ap}_{\Delta\left(\kappa(n, a), \beta_{\mathrm{B}_{\infty}}(n, a), \iota(k, b)\right) \#_{\mathrm{E}}}\left(\operatorname{apd}_{\mathrm{g}(n+1, \mathrm{a}(n, a))}(\kappa(k, b))\right)
\end{aligned}
$$

Figure 5. Our new goal 


$$
\begin{aligned}
\mathrm{H}(n, a, k+1, b):= \\
\quad \varepsilon\left(\kappa(k, b), \kappa(k+1, b), \beta_{\mathrm{c}_{\infty}}(n, a, k, b), \mathrm{h}(n+1, \mathrm{a}(n, a), k+1, \mathrm{c}(n, a, k+1, b))\right) . \\
\quad \operatorname{ap}_{\Delta\left(\kappa(n, a), \beta_{\mathrm{B}_{\infty}}(n, a), \iota(k, b)\right) \#_{\mathrm{E}}}(\mathrm{H}(n+1, \mathrm{a}(n, a), k, b))
\end{aligned}
$$

Figure 6. Definition of $\mathrm{H}(n, a, k+1, b)$

To define $\mathrm{H}(n, a, 0, b)$, we need a path

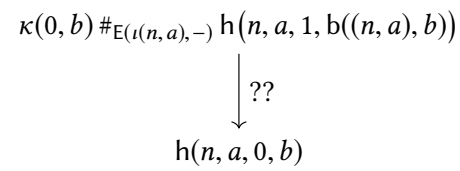

The obvious path to include is the assumption $\mathrm{p}(n, a, b)$, which has the correct right endpoint:

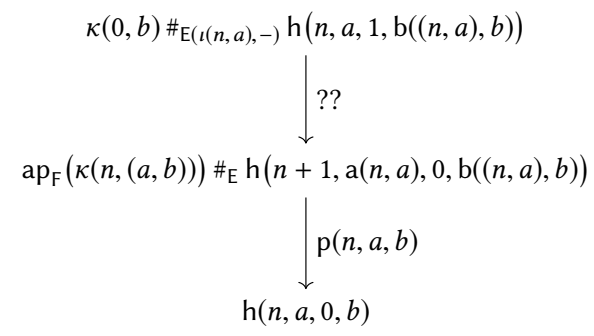

To fill the remaining edge, we define a a homotopy

- $\eta(\alpha, \beta, \theta): \beta \#_{\mathrm{E}(\iota(n, a),-)} \gamma \#_{\mathrm{E}} \sim \alpha \#_{\mathrm{E}}$

by induction on $\alpha, \beta, \theta$, where

- $y_{1}, y_{2}: \mathrm{C}_{\infty}(n, a)$ and $\alpha: y_{1}=y_{2}$

- $z: \sum_{x: \mathrm{A}_{\infty}} \mathrm{B}_{\infty}(x)$

- $\beta: z=\left(\iota(n, a), y_{1}\right)$

- $\gamma: z=\left(\iota(n, a), y_{2}\right)$

- $\theta: \gamma=\beta \cdot \operatorname{ap}_{(\iota(n, a),-)}(\alpha)$

This leads us to the path in Figure 7, where $\beta_{\mathrm{F}}(n,(a, b))$ is the witness for the second computation rule for $F$, expressing $\operatorname{ap}_{\mathrm{F}}(\kappa(n,(a, b)))$ as the composition

$$
\Delta\left(\kappa(n, a), \beta_{\mathrm{B}_{\infty}}(n, a), \iota(0, \mathrm{~b}((n, a), b))\right) \cdot \operatorname{ap}_{(\iota(n, a),-)}(\kappa(0, b))
$$

This finishes the construction of $\mathrm{H}$ and the definition of g. The equality in Figure 5 is now immediate by the computation rules for $g$ and Figure 6, and the construction of $G$ is thus complete.

\subsection{The computation rules}

The computation rule on points is obvious. Now we just need to show

$$
\operatorname{apd}_{\mathrm{G}}\left(\operatorname{ap}_{\mathrm{F}}(\kappa(n,(a, b)))\right)=\mathrm{p}(n, a, b)
$$

As we observed above, the witness $\beta_{\mathrm{F}}(n, a, b)$ expresses the path $\operatorname{ap}_{\mathrm{F}}(\kappa(n,(a, b)))$ as the composition

$$
\Delta\left(\kappa(n, a), \beta_{\mathrm{B}_{\infty}}(n, a), \iota(0, \mathrm{~b}((n, a), b))\right) \cdot \operatorname{ap}_{(\iota(n, a),-)}(\kappa(0, b))
$$

We would analogously like to express the dependent action of $\mathrm{G}$ on this composite path in terms of dependent actions on the individual paths. So we utilize the generalization we used earlier when defining $\eta$ : given

- $y_{1}, y_{2}: \mathrm{C}_{\infty}(n, a)$ and $\alpha: y_{1}=y_{2}$

- $z: \sum_{x: \mathrm{A}_{\infty}} \mathrm{B}_{\infty}(x)$

- $\beta: z=\left(l(n, a), y_{1}\right)$

- $\gamma: z=\left(\iota(n, a), y_{2}\right)$

- $\theta: \gamma=\beta \cdot \operatorname{ap}_{(\iota(n, a),-)}(\alpha)$

the path $\operatorname{apd}_{\mathrm{g}(n, a)}(\alpha)$ is equal to

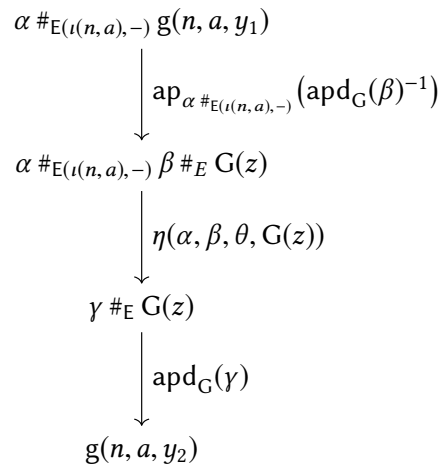

This is again immediate by path induction on $\alpha, \beta, \theta$. Instantiating this generalization thus expresses $\operatorname{apd}_{\mathrm{g}(n, a)}(\kappa(0, b))$ as the path in Figure 8. But by the second computation rule for $\mathrm{g}(n, a)$, we have $\operatorname{apd}_{\mathrm{g}(n, a)}(\kappa(0, b))=\mathrm{H}(n, a, 0, b)$. Comparing Figures 7 and 8 , it only remains to show that the path

$$
\operatorname{apd}_{\mathrm{G}}\left(\Delta\left(\kappa(n, a), \mathrm{c}_{\star}(n, a), \iota(0, \mathrm{~b}((n, a), b))\right)\right)^{-1}
$$

is equal to $\mu(n, a, 0, b)$, which is reflexivity. We use one last generalization: given

- $x_{1}, x_{2}: \mathrm{A}_{\infty}$ and $\alpha: x_{1}=x_{2}$

- $F: \mathrm{B}_{\infty}\left(x_{1}\right) \rightarrow \mathrm{B}_{\infty}\left(x_{2}\right)$

- $F_{\star}: \overline{\operatorname{ap}_{\mathrm{B}_{\infty}}(\alpha)}=F$

- $y: \mathrm{B}_{\infty}\left(x_{1}\right)$

- $w: \Pi_{y: \mathrm{B}_{\infty}\left(x_{1}\right)} \mathrm{G}\left(x_{2}, F(y)\right)=\Delta\left(\alpha, F_{\star}, y\right) \#_{E} \mathrm{G}\left(x_{1}, y\right)$

- $w_{\star}: \mathrm{I}\left(\alpha, F_{\star}, \mathrm{G}\left(x_{1},-\right), \mathrm{G}\left(x_{2},-\right)\right) \operatorname{apd}_{x, y \mapsto \mathrm{G}(x, y)}(\alpha)=w$

then $\operatorname{apd}_{\mathrm{G}}\left(\Delta\left(\alpha, F_{\star}, y\right)\right)^{-1}=w(y)$. We use the instantiation $w:=\omega(n, a)$, for which $w_{\star}$ follows by the second computation rule of the curried version of $\mathrm{G}$. This completes the proof. 


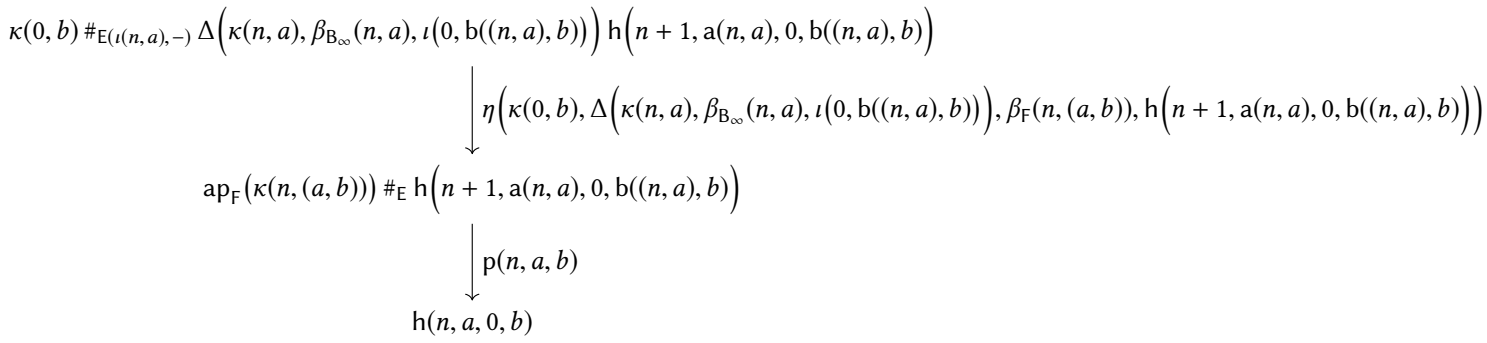

Figure 7. Definition of $\mathrm{H}(n, a, 0, b)$

$$
\begin{aligned}
& \kappa(0, b) \#_{\mathrm{E}(\iota(n, a),-)} \mathrm{h}(n, a, 1, \mathrm{~b}((n, a), b)) \\
& \operatorname{ap}_{\kappa(0, b) \#_{\mathrm{E}(\iota(n, a),-)}}\left(\operatorname{apd}_{\mathrm{G}}\left(\Delta\left(\kappa(n, a), \beta_{\mathrm{B}_{\infty}}(n, a), \iota(0, \mathrm{~b}((n, a), b))\right)\right)^{-1}\right) \\
& \kappa(0, b) \#_{\mathrm{E}(\iota(n, a),-)} \Delta\left(\kappa(n, a), \beta_{\mathrm{B}_{\infty}}(n, a), \iota(0, \mathrm{~b}((n, a), b))\right) \mathrm{h}(n+1, \mathrm{a}(n, a), 0, \mathrm{~b}((n, a), b)) \\
& \eta\left(\kappa(0, b), \Delta\left(\kappa(n, a), \beta_{\mathrm{B}_{\infty}}(n, a), \iota(0, \mathrm{~b}((n, a), b))\right), \beta_{\mathrm{F}}(n,(a, b)), \mathrm{h}(n+1, \mathrm{a}(n, a), 0, \mathrm{~b}((n, a), b))\right) \\
& \operatorname{ap}_{\mathrm{F}}(\kappa(n,(a, b))) \#_{\mathrm{E}} \mathrm{h}(n+1, \mathrm{a}(n, a), 0, \mathrm{~b}((n, a), b)) \\
& \operatorname{apd}_{\mathrm{G}}\left(\operatorname{ap}_{\mathrm{F}}(\kappa(n,(a, b)))\right) \\
& \mathrm{h}(n, a, 0, b)
\end{aligned}
$$

Figure 8. Characterization of $\operatorname{apd}_{\mathrm{g}(n, a)}(\kappa(0, b))$

\section{Applications of the Main Theorem}

We now establish some corollaries of our main result. Before we proceed, we first show that we can describe the colimit $\mathrm{B}_{\infty}(\iota(n, a))$ in a more explicit fashion that is often easier to work with.

Lemma 7.1. Given a sequence $(\mathrm{B}, \mathrm{b})$ fibered over $(\mathrm{A}, \mathrm{a})$ and $a: \mathrm{A}(n)$, we have

$$
\mathrm{B}_{\infty}(l(n, a)) \simeq \operatorname{colim}\left(k \mapsto \mathrm{B}\left((n, a)^{+k}\right), k \mapsto \mathrm{b}\left((n, a)^{+k}\right)\right)
$$

Proof. This follows from the definition

$$
\mathrm{B}_{\infty}(\iota(n, a)) \equiv \operatorname{colim}(k \mapsto \mathrm{C}(n, a, k), k \mapsto \mathrm{c}(n, a, k))
$$

and the functoriality of the colimit, using the fact that lifting is associative up to typal equality.

The first application of our theorem is the characterization of the path types of sequential colimits. We will need the following two lemmas, the proofs of which are straightforward:

Lemma 7.2. We have $\operatorname{colim}_{k}(1) \simeq 1$.

We will have a more general result in Corollary 7.7.5 but in that proof we will use a special case of this lemma.

Lemma 7.3 (Encode-decode). Let $a: A$ and let $B: A \rightarrow U$ be a type family with $b: B(a)$. Then the following are equivalent:
1. The canonical family of maps $\Pi_{x: A}(a=x) \rightarrow B(x)$ is $a$ family of equivalences.

2. The total space of $B$ is contractible.

We can now prove the following:

Theorem 7.4. Fix a sequence $(\mathrm{A}, \mathrm{a})$. For any $a_{1}, a_{2}: \mathrm{A}(0)$, we have an equivalence

$$
\left(\iota\left(0, a_{1}\right)=\mathrm{A}_{\infty} \iota\left(0, a_{2}\right)\right) \simeq \operatorname{colim}_{k}\left(a_{1}^{+k}=\mathrm{A}_{\mathrm{A}(k)} a_{2}^{+k}\right)
$$

and for any $a_{1}, a_{2}: \mathrm{A}(n)$, we have an equivalence

$$
\left(\iota\left(n, a_{1}\right)=\mathrm{A}_{\infty} \iota\left(n, a_{2}\right)\right) \simeq \operatorname{colim}_{k}\left(a_{1}^{+k}={ }_{\mathrm{A}(n+k)} a_{2}^{+k}\right)
$$

Proof. By Lemma 3.7 and the definition of the lifting $(-)^{+k}$ : $\mathrm{A}(n) \rightarrow \mathrm{A}(n+k)$, the latter equivalence immediately follows from the former. To prove the former, we define a fibered sequence $(B, b)$ over $(A, a)$ by

- $\mathrm{B}(k, a):=\left(a_{1}^{+k}=\mathrm{A}(k) a\right)$

- $\mathrm{b}(k, a, p):=\operatorname{ap}_{\mathrm{a}(k)}(p)$

Now we use Theorem 5.1 to deduce that the total space of $\mathrm{B}_{\infty}$ is contractible.

$$
\begin{aligned}
\sum_{x: \mathrm{A}_{\infty}} \mathrm{B}_{\infty}(x) & \simeq \operatorname{colim}_{k}\left(\sum_{a: \mathrm{A}(k)}\left(a_{1}^{+k}=\mathrm{A}_{\mathrm{A}(k)} a\right)\right) \\
& \simeq \operatorname{colim}_{k}(1) \\
& \simeq 1 .
\end{aligned}
$$


We have $\iota\left(0, \operatorname{refl}_{a_{1}}\right): \mathrm{B}_{\infty}\left(\iota\left(0, a_{1}\right)\right)$, so we can now conclude by Lemma 7.3 that

$$
\begin{aligned}
& \left(\iota\left(0, a_{1}\right)=\mathrm{A}_{\infty} \iota\left(0, a_{2}\right)\right) \\
\simeq & \mathrm{B}_{\infty}\left(\iota\left(0, a_{2}\right)\right) \\
\equiv & \operatorname{colim}\left(k \mapsto \mathrm{C}\left(0, a_{2}, k\right), k \mapsto \mathrm{c}\left(0, a_{2}, k\right)\right) \\
\simeq & \operatorname{colim}\left(k \mapsto \mathrm{B}\left(\left(0, a_{2}\right)^{+k}\right), k \mapsto \mathrm{b}\left(\left(0, a_{2}\right)^{+k}\right)\right) \\
\simeq & \operatorname{colim}\left(k \mapsto \mathrm{B}\left(k, a_{2}^{+k}\right), k \mapsto \mathrm{b}\left(k, a_{2}^{+k}\right)\right)
\end{aligned}
$$

where the second equivalence is Lemma 7.1 and the third is functoriality of the colimit.

Our next corollary shows that sequential colimits commute with loop spaces of pointed types. This proves Conjecture 3.8 of [Cavallo 2015] and answers Question 4.2 of [Graham 2017].

A pointed type $A$ is a pair consisting of a type, usually also denoted $A$, and a basepoint $a: A$. A pointed map $f: A \rightarrow^{*} B$ between types pointed with $a: A$ and $b: B$, respectively, is a pair consisting of a map $f: A \rightarrow B$ and a path $\beta: f(a)=b$.

The loop space $\Omega A$ of a pointed type $A$ is a pointed type with underlying type $a=a$ and basepoint $\operatorname{refl}_{a}$. A pointed map $f: A \rightarrow^{*} B$ induces a pointed map $\Omega f: \Omega A \rightarrow^{*} \Omega B$ whose underlying map is defined by induction on $\beta$ and the mapping $p: a=a$ to $\operatorname{ap}_{f}(p)$.

A pointed sequence is a sequence where all types and maps are pointed. We will denote the basepoint of $A_{n}$ by $a_{n}$ and now the sequential colimit is pointed with basepoint $\iota\left(0, a_{0}\right)$.

Corollary 7.5. For a pointed sequence $(\mathrm{A}, \mathrm{a})$ we have the following pointed equivalence

$$
\Omega A_{\infty} \simeq \operatorname{colim}(n \mapsto \Omega \mathrm{A}(n), n \mapsto \Omega \mathrm{a}(n)) .
$$

Proof. We compute

$$
\begin{aligned}
\Omega A_{\infty} & \simeq \operatorname{colim}_{n}\left(a_{0}^{+n}=a_{0}^{+n}\right) \\
& \simeq \operatorname{colim}_{n}\left(a_{n}=a_{n}\right)
\end{aligned}
$$

where the first equivalence is Theorem 7.4 and second is functoriality of the colimit. The fact that this equivalence is pointed is by reflexivity.

Given a natural transformation $\tau:\left(\mathrm{A}^{\prime}, \mathrm{a}^{\prime}\right) \rightarrow(\mathrm{A}, \mathrm{a})$ we can define the homotopy fiber of $\tau$ as a sequence fibered $\operatorname{over}(\mathrm{A}, \mathrm{a})$, by $\operatorname{fib}_{\tau}(n, a):=\operatorname{fib}_{\tau(n)}(a)$. Then the following corollary of the main theorem states that colimits commute with homotopy fibers.

Corollary 7.6. For a natural transformation $\tau:\left(\mathrm{A}^{\prime}, \mathrm{a}^{\prime}\right) \rightarrow$ $(\mathrm{A}, \mathrm{a})$ and a point $x: \mathrm{A}_{\infty}$, we have

$$
\mathrm{fib}_{\tau_{\infty}}(x) \simeq\left(\mathrm{fib}_{\tau}\right)_{\infty}(x) .
$$

Proof. Consider the following diagram, where the equivalences on the top are given by Theorem 5.1 (left) and by functoriality of the colimit together with the fact that the total space of the fiber of a function is the domain of that function (right).

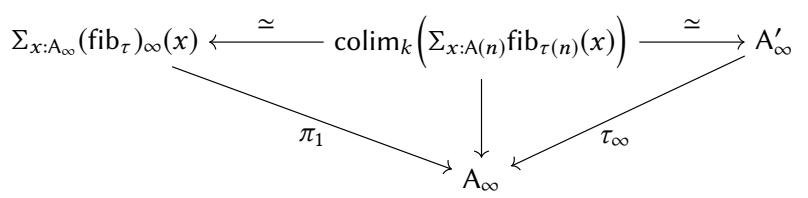

In this diagram the map in the middle is as given in Definition 4.3. The left triangle commutes by Theorem 5.1 and the right triangle commutes by functoriality of the colimit. Therefore,

$$
\mathrm{fib}_{\tau_{\infty}}(x) \simeq \mathrm{fib}_{\pi_{1}}(x) \simeq\left(\mathrm{fib}_{\tau}\right)_{\infty}(x)
$$

The following corollary specifies the interaction of colimits and truncations, truncatedness and connectedness. In particular, we show that colimits of $n$-truncated types are again $n$-truncated. In Theorem 3.3 of [Graham 2017], the definition of a homology theory is given as the set-truncation of a colimit of stable homotopy groups. Our result shows that this set-truncation is superfluous: the colimit is already a set.

Corollary 7.7. Consider a sequence (A, a) and some $k \geq-2$.

1. If each $\mathrm{A}(n)$ is $k$-truncated, then $\mathrm{A}_{\infty}$ is $k$-truncated.

2. We have an equivalence

$$
\left\|\mathrm{A}_{\infty}\right\|_{k} \simeq \operatorname{colim}_{n}\left(\|\mathrm{~A}(n)\|_{k},\|\mathrm{a}(n)\|_{k}\right) .
$$

3. If each $\mathrm{A}(n)$ is $k$-connected, then $\mathrm{A}_{\infty}$ is $k$-connected.

4. If $(\tau, H):(\mathrm{A}, \mathrm{a}) \rightarrow\left(\mathrm{A}^{\prime}, \mathrm{a}^{\prime}\right)$ is a natural transformation such that each $\tau(n)$ is $k$-truncated ( $k$-connected), then $\tau_{\infty}$ is $k$-truncated ( $k$-connected).

5. If each $\mathrm{a}(n)$ is $k$-truncated ( $k$-connected), then $\iota(0)$ is $k$-truncated ( $k$-connected).

Remark. By Lemma 3.7 we can generalize the quantification "for all $n: \mathbb{N}$ " in this Corollary to the weaker "there is an $m: \mathbb{N}$ such that for all $n \geq m$ ". In part 5 the conclusion then becomes that $\iota(m)$ is $k$-truncated ( $k$-connected).

Proof (of Corollary 7.7).

1. We prove this by induction on $k$.

The base case $k:=-2$ follows from Lemma 7.2 and the functoriality of the colimit.

For the successor case, take $x_{1}, x_{2}: \mathrm{A}_{\infty}$. We need to show that the type $x_{1}=\mathrm{A}_{\infty} x_{2}$ is $k$-truncated. We proceed by induction on $x_{1}$. Since being truncated is a mere proposition, we can assume that $x_{1}$ arises as a canonical point $\iota\left(n, a_{1}\right)$. We thus want to prove that for all $x_{2}: \mathrm{A}_{\infty}$, the type $\iota\left(n, a_{1}\right)={ }_{\mathrm{A}_{\infty}} x_{2}$ is $k$-truncated. Equivalences preserve truncatedness, so by Lemma 3.7 it suffices to prove that the type $\iota\left(0, a_{1}\right)=\mathrm{A}_{\infty}^{\prime} x_{2}$ is $k$ truncated for all $x_{2}: \mathrm{A}_{\infty}^{\prime}$, where $\mathrm{A}_{\infty}^{\prime}$ is the colimit of the sequence $\left(\mathrm{A}^{\prime}, \mathrm{a}^{\prime}\right):=(l \mapsto \mathrm{A}(n+l), l \mapsto \mathrm{a}(n+l))$. We proceed by induction on $x_{2}$, assuming it arises as 
a canonical point $\iota\left(m, a_{2}\right)$. Now we perform induction on $m$.

For the base case $m:=0$, we need to show that the type $\iota\left(0, a_{1}\right)={ }_{\mathrm{A}_{\infty}^{\prime}} \iota\left(0, a_{2}\right)$ is $k$-truncated. By Theorem 7.4 , it suffices to show that the type $\operatorname{colim}_{k}\left(a_{1}^{+k}={ }_{\mathrm{A}(k)} a_{2}^{+k}\right)$ is $k$-truncated. This follows from the induction hypothesis on $k$ and the fact that $\mathrm{A}(k)$ is $(k+1)$-truncated.

For the successor case, we need to show that the type $\iota\left(0, a_{1}\right)={ }_{\mathrm{A}_{\infty}^{\prime}} l\left(m+1, a_{2}\right)$ is $k$-truncated. Since we have the path $\kappa\left(0, a_{1}\right): \iota\left(1, \mathrm{a}\left(n, a_{1}\right)\right)=\mathrm{A}_{\infty}^{\prime} \iota\left(0, a_{1}\right)$, it suffices to show that the type $\iota\left(1, \mathrm{a}\left(n, a_{1}\right)\right)=\mathrm{A}_{\infty}^{\prime} l\left(m+1, a_{2}\right)$ is $k$-truncated. By Lemma 3.6, it suffices to show that the type $\iota\left(0, \mathrm{a}\left(n, a_{1}\right)\right)=\mathrm{A}_{\infty}^{\prime \prime} l\left(m, a_{2}\right)$ is $k$-truncated, where $\mathrm{A}_{\infty}^{\prime \prime}$ is the colimit of the sequence $\left(\mathrm{A}^{\prime \prime}, \mathrm{a}^{\prime \prime}\right):=(l \mapsto$ $\left.\mathrm{A}^{\prime}(l+1), l \mapsto \mathrm{a}^{\prime}(l+1)\right)$. But this follows from the induction hypothesis on $m$.

2. By the functoriality of the sequential colimit, we have a map $\mathrm{A}_{\infty} \rightarrow \operatorname{colim}_{n}\left(\|\mathrm{~A}(n)\|_{k},\|\mathrm{a}(n)\|_{k}\right)$. Because the right hand side is $k$-truncated, this induces a map

$$
g:\left\|\mathrm{A}_{\infty}\right\|_{k} \rightarrow \operatorname{colim}_{n}\left(\|\mathrm{~A}(n)\|_{k},\|\mathrm{a}(n)\|_{k}\right)
$$

For the other direction, we define a map

$$
h: \operatorname{colim}_{n}\left(\|\mathrm{~A}(n)\|_{k},\|\mathrm{a}(n)\|_{k}\right) \rightarrow\left\|\mathrm{A}_{\infty}\right\|_{k}
$$

by

$$
\begin{aligned}
& \iota\left(n,|a|_{k}\right) \mapsto|\iota(n, a)|_{k} \\
& \kappa\left(n,|a|_{k}\right) \mapsto \operatorname{ap}_{|-|_{k}}(\kappa(n, a))
\end{aligned}
$$

It is straightforward to show that both $h \circ g$ and $g \circ h$ are homotopic to the identity.

3. Since $\mathrm{A}(n)$ is $k$-connected, $\|\mathrm{A}(n)\|_{k}$ is contractible, so $\mathrm{b}\left\|\mathrm{A}_{\infty}\right\|_{k}$ is contractible by parts 1 and 2 .

4. We need to show that for any $x: \mathrm{A}_{\infty}^{\prime}$, the type $\mathrm{fib}_{\tau_{\infty}}(x)$ is $k$-truncated ( $k$-connected). This is a mere proposition, so we may assume that $x$ arises as a canonical point $\iota(n, a)$. Now

$$
\mathrm{fib}_{\tau_{\infty}}(\iota(n, a)) \simeq\left(\mathrm{fib}_{\tau}\right)_{\infty}(\iota(n, a))
$$

by Corollary 7.6. Since $\operatorname{fib}_{\tau(n)}(x)$ is $k$-truncated $(k$ connected) for all $n$ and $x$, we know that its colimit $\left(\mathrm{fib}_{\tau}\right)_{\infty}(l(n, a))$ is $k$-truncated $(k$-connected), by part 1 (3).

5. Consider the natural transformation from the constant sequence $k \mapsto \mathrm{A}(0)$ to $(\mathrm{A}, \mathrm{a})$ below:

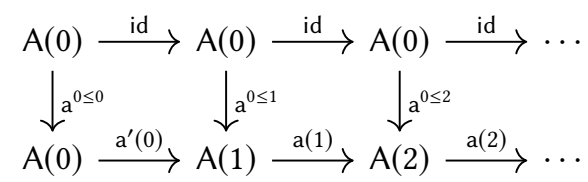

The maps $\mathrm{a}^{0 \leq n}$ are defined by $\mathrm{a}^{0 \leq 0}:=$ id and $\mathrm{a}^{0 \leq n+1}:=$ $\mathrm{a}(n) \circ \mathrm{a}^{0 \leq n}$. Each $\mathrm{a}^{0 \leq n}$ is $k$-truncated ( $k$-connected). Therefore, by part 4 the map $\mathrm{a}^{0 \leq \infty}: \operatorname{colim}_{n}(\mathrm{~A}(0)) \rightarrow$
$\mathrm{A}_{\infty}$ is $k$-truncated ( $k$-connected). Composing $\mathrm{a}^{0 \leq \infty}$ and the canonical equivalence $\mathrm{A}(0) \simeq \operatorname{colim}_{n}(\mathrm{~A}(0))$ yields precisely $\iota(0)$, which is thus $k$-truncated ( $k$-connected) as well.

Acknowledgements. We thank Alex Kavvos for his careful reading of this paper and helpful discussions and feedback, including suggestions for future work. We also thank the anonymous referees. The first author gratefully acknowledges the support of the National Science Foundation through grant CNS-1704788. Any opinions, findings, and conclusions or recommendations expressed in this material are those of the author(s) and do not necessarily reflect the views of the National Science Foundation. The second author gratefully acknowledges the support of the Air Force Office of Scientific Research through MURI grant FA9550-15-1-0053. The third author gratefully acknowledges the support of the Air Force Office of Scientific Research through grant FA9550-17-1-0326. Any opinions, findings and conclusions or recommendations expressed in this material are those of the authors and do not necessarily reflect the views of the AFOSR.

\section{References}

J. Adamek. 1974. Free Algebras and Automata Realizations in The Language of Categories. In Commentationes Mathematicae Universitatis Carolinae, Vol. 15. 589-604.

S. Awodey, R. Garner, P. Martin-Löf, and V. Voevodsky (organizers). 2011. Mini-Workshop (Oberwolfach): The Homotopy Interpretation of Constructive Type Theory. (2011). Available at https://hottheory.files. wordpress.com/2011/06/report-11_2011.pdf.

S. Awodey and M. Warren. 2009. Homotopy-Theoretic Models of Identity Types. Mathematical Proceedings of the Cambridge Philosophical Society 146, 1 (2009), 45-55.

H. Barendregt. 1985. The Lambda Calculus - Its Syntax And Semantics. Studies in Logic and the Foundations of Mathematics, Vol. 103. North-Holland.

F. Borceux. 1994. Handbook of Categorical Algebra. Cambridge University Press.

G. Brunerie. 2016. On the Homotopy Groups of Spheres in Homotopy Type Theory. (2016). Available at arxiv.org as arXiv:1606.05916.

E. Cavallo. 2015. Synthetic Cohomology in Homotopy Type Theory. Master's thesis. Carnegie Mellon University.

J. Christensen, M. Opie, E. Rijke, and L. Scoccola. 2018. Localization in Homotopy Type Theory. (2018).

L. de Moura, S. Kong, J. Avigad, F. van Doorn, and J. von Raumer. 2015. The Lean Theorem Prover (System Description). Conference on Automated Deduction (CADE 2015) (2015), 378-388.

R. Graham. 2017. Synthetic Homology in Homotopy Type Theory. (2017). Available at arxiv.org as arXiv:1706.01540.

C. Kapulkin and P. LeFanu Lumsdaine. 2012. The Simplicial Model of Univalent Foundations (after Voevodsky). (2012). To appear in the fournal of European Mathematical Society. Available at arxiv.org as arXiv:1203.3253v5.

N. Kraus and J. von Raumer. 2019. Path Spaces of Higher Inductive Types in Homotopy Type Theory. In To appear in Logic in Computer Science (LICS 2019).

P. LeFanu Lumsdaine. 2011. Higher Inductive Types: a Tour of the Menagerie. (2011). Available at https://homotopytypetheory.org/2011/04/24/higherinductive-types-a-tour-of-the-menagerie/.

P. LeFanu Lumsdaine and M. Shulman. 2017. Semantics of Higher Inductive Types. (2017). Available at arxiv.org as arXiv:1705.07088v2. 
J. Lurie. 2009. Higher Topos Theory. Annals of Mathematics Studies, Vol. 170. Princeton University Press.

S. MacLane. 1971. Categories for the Working Mathematician. Graduate Texts in Mathematics, Vol. 5. Springer-Verlag.

P. Martin-Löf. 1975. An Intuitionistic Theory of Types: Predicative Part. In Logic Colloquium 1973. North-Holland.

E. Rijke. 2017. The Join Construction. (2017). Available at arxiv.org as arXiv:1701.07538.

M. Shulman. 2011. Homotopy Type Theory, VI. Available at https://golem.ph. utexas.edu/category/2011/04/homotopy_type_theory_vi.html. (2011).

M. Shulman. 2013. Spectral Sequences. Available at https:// homotopytypetheory.org/2013/08/08/spectral-sequences/. (2013).

M. Shulman. 2019. All ( $\infty, 1)$-Toposes Have Strict Univalent Universes. (2019). Available at arxiv.org as arXiv:1904.07004
K. Sojakova. 2016. Higher Inductive Types as Homotopy-Initial Algebras. Ph.D. Dissertation. Carnegie Mellon University.

The Univalent Foundations Program, Institute for Advanced Study. 2013. Homotopy Type Theory - Univalent Foundations of Mathematics. Univalent Foundations Project.

F. van Doorn. 2016. Constructing the Propositional Truncation Using NonRecursive HITs. In Certified Programs and Proofs (CPP 2016). 122-129.

V. Voevodsky. 2010. The Equivalence Axiom and Univalent Models of Type Theory. (2010). Invited talk at Carnegie Mellon University, available at arxiv.org as arXiv:1402.5556v2.

V. Voevodsky. 2011. Univalent Foundations of Mathematics. (2011). Invited talk at the Workshop on Logic, Language, Information and Computation (WoLLIC 2011). 